\title{
Poster Exhibit 2: Non-prostate Genitourinary Cancers
}

Cite as: Can Urol Assoc / 2021;15(6S2):S84-98. http://dx.doi.org/10.5489/cuaj.7401

\section{UP-27}

Uptake of a second transurethral resection of the bladder tumor in T1 bladder cancer in Ontario: An interrupted time series analysis involving 15 years of observation

Marian S. Wettstein ${ }^{1,2,3,4}$, Nancy N. Baxter ${ }^{2,3}$, Rinku Sutradhar ${ }^{2,3}$, Muhammad M. Mamdani ${ }^{2,3}$, Song Pham ${ }^{3}$, Syed R. Qadri', Kathy Li', Ning Liu', van der Kwast Theodorus ${ }^{5}$, Thomas Hermanns, Girish S. Kulkarni, ${ }^{1,2,3}$

'Division of Urology, Department of Surgery, Princess Margaret Cancer Centre, University Health Network, University of Toronto, Toronto, ON, Canada; ${ }^{2}$ Institute of Health Policy, Management and Evaluation, University of Toronto, Toronto, ON, Canada; ${ }^{3} \mathrm{ICES}$, Toronto, ON, Canada; ${ }^{4}$ Department of Urology, University Hospital of Zurich, University of Zurich, Zurich, Switzerland; ${ }^{5}$ Department of Pathology, Princess Margaret Cancer Centre, University Health Network, University of Toronto, Toronto, ON, Canada Introduction: A second transurethral resection of the bladder tumor (reTUR) within 2-6 weeks after initial resection is thought to have diagnostic, therapeutic, and prognostic benefits in T1 bladder cancer. However, little is known about the real-world uptake of this guideline-endorsed intervention. We aimed to: 1 ) measure reTUR rates over time; 2 ) investigate if a guideline revision (April 2008) explicitly endorsing reTUR within $2-6$ weeks in all T1 bladder cancer patients led to an increase in reTUR rates; and 3) investigate the uptake among different groups of surgeons.

Methods: Province-wide bladder cancer pathology reports (January 2001 to December 2015; Ontario, Canada) were manually abstracted and linked with health administrative data to: 1 ) identify primary cases of T1 bladder cancer and 2) ascertain whether these patients received reTUR. The resulting patients were then aggregated into quarterly time series and investigated by descriptive analysis, ARIMA modeling, and Poisson regression analysis. Results: A cohort of 7373 patients was aggregated into a time series. We observed a linear increase in reTUR rates from $8.4 \%$ in 2001 to $28.3 \%$ in 2015. An actual effect of the guideline revision in April 2008 on reTUR rates could not be detected $(p=0.41)$. However, we observed a rather heterogeneous uptake behavior among different groups of surgeons. Specifically, female surgeons, more junior surgeons, high-volume surgeons, Canadian graduates, and surgeons without an academic affiliation were all independently more likely to perform reTUR (all $\mathrm{p}<0.05$ in adjusted analysis).

Conclusions: ReTUR rates in primary T1 bladder cancer increased between 2001 and 2015 in Ontario regardless of the guideline revision in April 2008. Our study demonstrates that the uptake of this guideline-endorsed intervention varies among different groups of surgeons and therefore warrants further research to identify barriers to change that can be addressed by tailored interventions.

\section{UP-28}

Adherence to guidelines in the management of high-risk nonmuscle-invasive bladder cancer: Are patients receiving intravesical Bacillus Calmette-Guérin therapy appropriately?

Betty Wang ${ }^{1}$, Benjamin Beech ${ }^{7}$, Sentil Senthilselvan ${ }^{2}$, Howard J. Evans ${ }^{1}$

${ }^{1}$ Division of Urology, Department of Surgery, University of Alberta, Edmonton, $\mathrm{AB}$, Canada; ${ }^{2} \mathrm{~S}$ chool of Public Health, University of Alberta, Edmonton, $\mathrm{AB}$, Canada

Introduction: High-risk non-muscle-invasive bladder cancer (HR-NMIBC) is defined as all tumors with T1 stage, carcinoma in situ (CIS), or high grade (HG). In this setting, Bacillus Calmette-Guérin (BCG) intravesical therapy is the standard of care, which comprises of both an induction course over six weeks, with a maintenance course over three years. We aim to deter- mine the adherence to this level $1 \mathrm{~A}$ recommendation in our center, and to identify predictors associated with BCG use.

Methods: We performed a retrospective review of patients receiving transurethral resection of bladder tumors (TURBT) between November 2009 and November 2014. Patients were included if they had HR-NMIBC. We determined whether these patients received BCG and if so, the duration of treatment. We also collected relevant clinical variables that were felt could potentially influence the use of BCG. These included patient factors, such as age and gender, as well as disease factors, such as stage, grade, presence of variant histology, and history of prior interventions. We performed a multivariable analysis using logistic regression models to identify any correlation between BCG use and any of the clinical variables.

Results: A total of 608 patients were identified as having HR-NMIBC; $76 \%$ of patients had no prior surgery for their bladder cancer and $91 \%$ of patients never received prior BCG. Of all patients with HR-NMIBC, 59\% of patients received $B C G$. Using multivariate logistic regression models, age, T-stage, and surgeon volume were associated with BCG therapy. Number of prior TURBTs and history of prior BCG therapy was not associated with BCG therapy.

Conclusions: In summary, data showed that $59 \%$ of patients with HR-NMIBC received some duration of BCG. Age, T-stage, and surgeon were identified as predictors of BCG therapy. Given this data, we have identified a gap for future quality improvement. More work is needed to help improve uptake of BCG therapy in the clinically appropriate patient.

\section{UP-29}

Safety and efficacy of transurethral resection of bladder tumor comparing spinal anesthesia to spinal anesthesia with an obturator nerve block: A systematic review and meta-analysis

Anil Krishan ${ }^{1}$, Angus Bruce', Shehab Khashaba², Mohamed Abouelela3, Syed A Ehsanullah⿱

${ }^{1}$ Urology, Walsall Manor Hospital, Walsall, United Kingdom; ${ }^{2}$ Urology, King Hamad University Hospital, Busaiteen, Bahrain; ${ }^{3}$ Anesthesia, King Hamad University Hospital, Busaiteen, Bahrain; ${ }^{4}$ Urology, University Hospitals Birmingham, Birmingham, United Kingdom

Introduction: We aimed to investigate whether spinal anesthesia with an obturator nerve block (SA+ONB) can be effectively employed for transurethral resection of bladder tumors (TURBT) during the COVID-19 pandemic to improve patient outcomes, while also avoiding aerosol-generating procedures. Our goal was to compare outcomes of TURBT using spinal anesthesia (SA) alone vs. SA+ONB in terms of rates of obturator reflex, bladder perforation, incomplete tumor resection, tumor recurrence, and local anesthetic toxicity.

Methods: We conducted a comprehensive search of electronic databases, identifying studies comparing the outcomes of TURBT using SA vs. SA+ONB. The Cochrane risk-of-bias tool for randomized controlled trials (RCTs) and the Newcastle-Ottawa scale for observational studies were used to assess the included studies. Random effects modelling was used to calculate pooled outcome data.

Results: Four RCTs and three cohort studies were identified, enrolling 448 patients. The use of $\mathrm{SA}+\mathrm{ONB}$ was associated with a significantly reduced risk of obturator reflex $(p<0.00001)$, bladder perforation $(p=0.02)$, incomplete resection $(p<0.0001)$, and 12 -month tumor recurrence $(p=0.005)$. ONB was not associated with an increased risk of local anesthetic toxicity $(0 / 159)$.

Conclusions: Our meta-analysis suggests that TURBT employing SA+ONB is superior to the use of SA alone. During the COVID-19 pandemic, where 
avoidance of aerosol-generating procedures such as a general anesthesia is paramount, the use of an ONB with SA is essential for the safety of both patients and staff without compromising care. Further high-quality RCTs with adequate sample sizes are required to compare the different techniques of ONB, as well as comparing this method to general anesthesia with complete neuromuscular blockade.

\section{UP-30 \\ Impaired emotional, physical, and social well-being in radical cystectomy patients}

Iulie G. Trudel ${ }^{1}$, Conrad Maciejewski

'The Ottawa Hospital Research Institute, The Ottawa Hospital, Ottawa, ON, Canada; ${ }^{2}$ Department of Surgery, Division of Urology, The Ottawa Hospital, Ottawa, ON, Canada

Introduction: Radical cystectomy (RC) can have a negative impact on the patient's emotional, physical, and social well-being along the illness trajectory. There is a paucity of studies examining the health-related quality of life (HRQOL) of bladder cancer patients before RC. This study evaluated the HRQOL of RC patients before and after surgery.

Methods: RC patients were enrolled in the Diana Wood Cancer Survivorship Clinic at the Ottawa Hospital from January 2019. Questionnaires were completed prior to and 2-3 months post-RC. Validated HRQOL instruments were used: FACT-BI, SF-36 and Distress Thermometer (DT). Wilcoxon matched pairs signed Rank Tests were performed to determine changes in HRQOL scores.

Results: Fifty-four RC patients completed HRQOL measures before and after RC. Mean age was 67 years (standard deviation [SD] 9.7); 67\% were men and $61 \%$ underwent an ileal conduit diversion. Clinically significant pre-post differences were seen in medians for four SF-36 scale scores. Lower post-cystectomy scores were obtained in: physical functioning $(p=0.00)$, role limitations due to physical health $(p=0.00)$, energy/ fatigue $(p=0.01)$, and social well-being $(p=0.02)$. Clinically significant differences were also found for DT scores. Patients exhibited more emotional distress before RC $(p=0.005)$ and more physical concerns after RC $(p=0.007)$. FACT-BI results correspond with those obtained with SF-36 and DT. Moreover, after RC, younger patients ( $<65$ years) were less content with their social life and the older ones ( $\geq 65$ years) exhibited more fatigue. Neo-bladder patients cited more physical problems after RC, including urinary, sexual, gastrointestinal concerns and fatigue.

Conclusions: Emotional distress is often reported before RC and physical concerns are frequently identified after RC. Older patients and neo-bladder diversions encountered more physical problems after RC. Cancer survivorship needs in RC patients must be tailored to patients' age and urinary diversion to optimize HRQOL outcomes.

\section{UP-31}

Sex differences among those with bladder cancer postcystectomy: A retrospective, population-based study

\section{lain Maclntyre" ${ }^{1}$, D. Robert Siemens', Marlo Whitehead ${ }^{1}$}

${ }^{1}$ Urology, Queen's University, Kingston, ON, Canada; 'Institute for Clinical Evaluative Sciences, Queen's University, Kingston, ON, Canada

Introduction: Current dogma would suggest differential outcomes for women with bladder cancer post-cystectomy, although the evidence is controversial. The purpose of this study is to determine whether there is a difference in early or late outcomes between males and females among those who underwent radical cystectomy for bladder cancer.

Methods: This retrospective, population-based study reports on all patients that underwent radical cystectomy in Ontario using electronic records of treatment recorded in the ICES database. The primary outcome is to determine if both overall and cancer-specific survival differed between males and females among those who needed radical cystectomy upon initial presentation or those who progressed to needing radical cystectomy. Secondary outcomes include early postoperative mortality and readmission rates. A Cox proportional-hazards regression model was used to adjust for known confounders.

Results: There were 645 males and 248 females who underwent cystectomy for bladder cancer de novo; 538 males and 142 females were progressors. Analysis of these cases revealed that there were no sex differences in either overall survival (hazard ratio [HR] 1.16, 95\% confidence interval [Cl] 0.98-1.38) or cancer-specific survival (HR 1.14, 95\% Cl 0.95-1.38) among those who present with aggressive disease de novo. Similarly, there were no sex differences among those who progressed to cystectomy in both overall survival (HR 1.02, 95\% Cl 0.80-1.29) and cancer-specific survival (HR 1.06, $95 \% \mathrm{Cl}$ 0.81-1.39). Additionally, there were no sex-associated differences in mortality or readmission at either 30 or 90 days postoperatively.

Conclusions: Based on this review of all patients in Ontario undergoing cystectomy for bladder cancer, there were no clinically significant differences in early or late morbidity or mortality outcomes between men and women.

\section{UP-32}

Molecular subtyping to stratify the treatment of muscle-invasive bladder cancer: A cost-effectiveness analysis

Diana E. Magee ${ }^{1,2}$, Douglas C. Cheung ${ }^{1,2}$, Beate Sander ${ }^{2,3}$, Girish S. Kulkarni ${ }^{1,2}$

'Division of Urology, Department of Surgery, University Health Network, University of Toronto, Toronto, ON, Canada; ${ }^{2}$ Institute of Health Policy, Management and Evaluation, University of Toronto, Toronto, ON, Canada; ${ }^{3}$ Toronto Health Economics and Technology Assessment Collaborative (THETA), University of Toronto, Toronto, ON, Canada

Introduction: The gold standard treatment for muscle-invasive bladder cancer (MIBC) is neoadjuvant chemotherapy (NAC) followed by radical cystectomy. However, response to NAC is unpredictable. Molecular subtypes allow for an improved ability to select a tailored treatment course. Our study aims to assess the cost-effectiveness of molecular subtyping in the management of MIBC.

Methods: A two-dimensional Markov microsimulation model was developed using TreeAge Pro comparing three strategies: NAC at current usage rates $(36 \%)$, universal NAC usage, and molecular subtype-directed care. Model probabilities and utilities were derived from the published literature. Cost of each phase of care was obtained from primary data and the Canadian Institute for Health Information patient cost estimator. The primary outcomes were quality-adjusted life years (QALYs), cost, overall survival (OS), and the incremental cost-effectiveness ratio (ICER). Results: The predicted QALYs were 8.34, 8.73, and 9.14, with costs of $\$ 62478, \$ 76962$, and $\$ 62579$ for NAC at current usage rates, universal NAC usage, and subtype-directed care, respectively. OS at 10 years was $39.2 \%, 40.8 \%$, and $42.8 \%$ for NAC at current usage rates, universal NAC usage, and subtype-directed care, respectively. When comparing subtypedirected care to current rates of NAC usage, the ICER was \$127/QALY. Subtype-directed care dominated universal NAC usage.

Conclusion: We demonstrated that in patients with MIBC, a molecular subtype-directed approach to the administration of NAC can result in improved OS, greater QALYs, and be cost-effective within a single-payer healthcare system. A push to the universal use of NAC will result in improved survival compared with what our current rates of use achieve but is likely not the best approach considering the drawbacks of chemotherapy, including toxicity and unequal response. This model is built upon the available literature and requires validation prior to clinical implementation.

\section{UP-33}

The University of Alberta experience with radical cystectomy via minimally invasive approaches

Alexander Hengel ${ }^{1}$, Jan K. Rudzinski ${ }^{1,2}$, Benjamin Beech ${ }^{1}$, Natasha P. Govindasamy², Eric Estey', Howard J. Evans', Blair A. St Martin

${ }^{1}$ Division of Urology, University of Alberta, Edmonton, AB, Canada; ${ }^{2}$ Department of Oncology, University of Alberta, Edmonton, AB, Canada Introduction: Radical cystectomy is the standard of care treatment for muscle-invasive bladder cancer. It is associated with high morbidity and significant risk of peri- and postoperative complications. Minimally invasive (MI) approaches seek to lessen the morbidity without sacrificing oncological outcomes. Due to limited resources and the technical challenges involved, MI radical cystectomy (MIRC) has had limited uptake in Canada. Herein, we present, to our knowledge, the largest case series of MIRC in a Canadian setting. 
Methods: A retrospective review was performed. We identified all patients undergoing MIRC for bladder cancer at our center from 2006-2020. We collected data on demographics, oncological staging, peri- and postoperative outcomes, length of stay (LOS), perioperative blood transfusions, uretero-ileal anastomotic stricture occurrence, recurrence-free survival, and overall survival (OS). Descriptive statistics and Kaplan-Meir survival analysis were used to assess outcomes.

Results: Overall, 29 patients underwent MIRC, 10 using a laparoscopic approach and 19 using robotic-assisted laparoscopic approach. Median age at time of MIRC was 66.5 years. Ileal conduit urinary diversion was performed in 23/29, neobladder in 5/29, and one patient was left without urinary diversion (hemodialysis). Median LOS was eight days (range 3-89). Three patients received blood transfusions within 90 days of MIRC Uretero-ileal anastomotic stricture occurred in 6/28 (21.4\%). In total, seven patients had recurrence of their cancer, with the median time to recurrence of 9.8 months (range 4.6 months to 4.9 years). Predicted five-year OS of the group was $57.3 \%$ (confidence interval 37.2-73.1\%). Conclusions: We present the University of Alberta experience with MIRC for bladder cancer. Outcomes were modest and may reflect the learning curve of these technically challenging procedures. Further study is required to elucidate the role $\mathrm{MI}$ vs. open approach to radical cystectomy, especially in the resource-limited Canadian setting.

\section{UP-34}

Evaluation of financial toxicity in Canadian patients undergoing radical cystectomy

Ryan McLarty ${ }^{1}$, Julie Trudel ${ }^{1,2}$, Conrad Maciejewski ${ }^{1}$

'Division of Urology, Department of Surgery, University of Ottawa, Ottawa, ON, Canada; ${ }^{2}$ The Ottawa Hospital Research Institute, University of Ottawa, Ottawa, ON, Canada

Introduction: Financial toxicity (FT) describes the financial consequences of cancer and its treatment. It has been associated with worse patientreported outcomes, such as quality of life and symptom burden. FT in patients undergoing radical cystectomy $(\mathrm{RC})$ has not been well-examined. The objective of this study was to evaluate FT in RC patients.

Methods: Patients were recruited from the Diana Wood Cance Survivorship Clinic at The Ottawa Hospital between January 2019 and October 2020. The validated 11-item Comprehensive Score for Financial Toxicity (COST) questionnaire was administered twice: before RC and 2-3 months after RC. Higher scores (0-44) represent less FT and they are presented as medians. Wilcoxon matched pairs signed rank tests were used to compare pre-and post-RC COST scores.

Results: Fifty-four patients completed the COST questionnaire at both time points. The mean age was 67 years (standard deviation [SD] 9.7); 67\% were male and $61 \%$ underwent an ileal conduit urinary diversion. There was a clinically significant difference between pre- and post-RC COST scores, with lower post-RC scores ( $p=0.001$; medians: 37.2 vs. 30.4 ). This difference was maintained regardless of the patients' gender and type of urinary diversion $(p<0.05)$. As for the patients' age, a clinically significant difference in COST scores was only found for older patients ( $\geq 65$ years) ( $p=0.001$; medians: 38.8 vs. 32.0$)$. Younger patients' ( $<65$ years) pre-postCOST scores didn't differ significantly ( $p=0.28$; medians: 32.5 vs. 25.0 ). Conclusions: This is the first study to report COST scores for Canadian patients undergoing RC. RC patients experience more FT after surgery, except for younger patients, who tend to display more FT both before and after surgery. These findings provide useful information for implementing patient-centered financial assessment and counselling in patients under- going RC. Further studies are required to better understand FT after RC and in younger patients.

\section{UP-35}

Visual prediction of urothelial cell carcinoma grade and stage: A prospective clinical trial

Heather Morris' ${ }^{\prime}$, Ross Mason', Gregory G. Bailly', David G. Bell', Ashley R. Cox', Jon Duplisea', Ricardo A. Rendon', Thomas A. Skinner', Peter Massaro

${ }^{1}$ Urology, Dalhousie University, Halifax, NS, Canada

Introduction: Urothelial cell carcinoma (UC) is most commonly diagnosed via cystoscopy, followed by transurethral resection of the bladder tumor (TURBT). At the time of TURBT, intravesical instillation of chemotherapy is a recommended treatment for patients felt to have solitary low-grade lesions. ${ }^{1,2}$ This requires the urologist to make assumptions about the pathology results before histological confirmation. Few studies have examined the accuracy of urologists' predictions on bladder tumor histology based on its endoscopic appearance. ${ }^{1,3,4}$ We sought to study the accuracy of urologists' predictions of grade, stage, and presence of carcinoma in situ (CIS) at the time of initial TURBT.

Methods: We performed a prospective clinical trial of patents with newly diagnosed bladder tumor(s) felt to be UC, who underwent TURBT. Urologists were asked to predict tumor stage, grade, and presence of CIS based on white light cystoscopy. They were not blinded to any preoperative evaluations. Predictions were compared to pathologist assessments. Results: Early results include predictions on 21 patients with a new diagnosis of a bladder tumor felt to be UC. Final pathology confirmed one lesion as solely CIS, 10 lesions as Ta, five lesions as T1, three lesions as $\mathrm{T} 2$, and two as benign lesions. The positive predictive value (PPV) and negative predictive value (NPV) for low-grade tumors were $28.6 \%$ and $75.0 \%$, respectively. For high-grade, the PPV and NPV were $85.7 \%$ and $57.1 \%$, respectively. For concurrent CIS, the PPV was $33.3 \%$ and the NPV was $100 \%$. Table 1 displays the full results.

Conclusions: Herein, we present early results from our study evaluating the accuracy of urologists in predicting the stage of newly diagnosed bladder tumors. Urologists are better at predicting high-grade tumors than they are at low-grade, as suggested by our predictive values. These findings have implications when selecting patients for immediate postoperative instillations and when triaging the urgency of postoperative followup.

\section{References}

1. Chang SS, Boorjian SA, Chou R et al. Diagnosis and treatment of non-muscle invasive bladder cancer: AUA/SUO guideline. J Urol 2016;196:1021. https://doi.org/10.1016/j.juro.2016.06.049

2. Kassouf W, Traboulsi SL, Kulkarni GS, et al. CUA guidelines on the management of non-muscle-invasive bladder cancer. Can Urol Assoc / 2015;9:E690-704. https://doi.org/10.5489/cuaj.3320

3. During VA, Sole GM, Jha AK, et al. Prediction of histological stage based on cystoscopic appearances of newly diagnosed bladder tumors. Ann R Coll Surg Engl 2016;98:547-51. https://doi. org/10.1308/rcsann.2016.0246

4. Marriappan P, Lavin V, Phua CQ, et al. Predicting grade and stage at cystoscopy in newly presenting bladder cancers - a prospective, double-blind clinical study. Urology 2017;109:134-9. https://doi. org/10.1016/j.urology.2017.08.007

UP-35. Table 1. Predictive values, specificities, and sensitivities of histology predictions

\begin{tabular}{lcccccc}
\hline & Low-grade & High-grade & Concurrent CIS & Stage TA & Stage T1 & Stage T2+ \\
\hline Positive predictive value & 28.6 & 85.7 & 33.3 & 81.8 & 33.3 & 0 \\
Negative predictive value & 75 & 57.1 & 100 & 90 & 80 & 84.2 \\
Sensitivity & 50 & 80 & 100 & 90 & 40 & 0 \\
Specificity & 35.3 & 66.7 & 21.1 & 81.8 & 75 & 88.9 \\
\hline
\end{tabular}




\section{UP-36}

The prognostic value of urinary cytology after trimodal therapy for muscle-invasive bladder cancer

Louise McLoughlin', Sophie O'Halloran ${ }^{1}$, Michael Tjong' ${ }^{2}$, Khaled Ajib', Katherine Lajkosz', Peter Chung' ${ }^{2}$, Neil E. Fleshner', Alejandro Berlin ${ }^{2}$ ${ }^{1}$ Department of Surgical Oncology (Urology), University Health Network, Toronto, ON, Canada; ${ }^{2}$ Department of Radiation Oncology, Princess Margaret Cancer Centre, Toronto, ON, Canada

Introduction: Complete response after trimodal therapy (TMT) for muscleinvasive bladder cancer (MIBC) includes a negative urine cytology (NC), no visible tumor, and negative biopsies. The significance of positive urinary cytology (PC) post-TMT (pTMT) without lesions in the upper tract or intravesical clinical/pathological recurrence is unknown. Altered cytology PTMT may represent recurrence, treatment effects, or a marker of de novo urothelial lesions. Rates and temporal trends of PC PTMT were analyzed to determine its potential use as a prognostic marker for bladder cancer recurrence pTMT.

Methods: This is a retrospective study of 150 patients who had TMT for MIBC at a single academic institution from 2002-2017. Available cytology results (NC vs. PC/equivocal) pTMT were evaluated for association with recurrence-free survival (RFS) $(n=128)$. Time to recurrence, stratified by first cytology pTMT, and time to NC were assessed by the Kaplan-Meier method. Differences between groups were evaluated with the log rank test. Results: We observed 61 recurrences in 128 patients with a median followup of 3.6 years (range $0.3-14.2)$ : $13(21 \%)$ local, $11(18 \%)$ urinary tract, $13(21 \%)$ metastasis, $24(39 \%)$ other. PC pTMT occurred in $41(32 \%)$ patients at first followup; NC occurred in $115(90 \%)$ patients at any point. Median RFS was shorter with PC vs. NC at first followup pTMT (21.3 vs. 78.1 months, $\mathrm{p}=0.047)$, with an increase in cumulative recurrence rate at 3.3 years $(\mathrm{PC} \mathrm{n}=24[60 \%]$ vs. $\mathrm{NC} \mathrm{n}=37[42 \%]$ $\mathrm{p}=0.085)$. Persistence of PC ( $\geq 4$ months pTMT, $\mathrm{n}=33,22 \%)$ had a median time to $\mathrm{NC}$ of 3.22 months (95\% confidence interval [CI] 2.99-5.80). Longer time to NC was associated with recurrence (3.68 months [95\% $\mathrm{Cl} 3.22-8.96]$ vs. 2.76 months [95\% Cl 0.69-2.99]).

Conclusions: NC post-TMT and shorter interval to NC is associated with improved RFS and potentially lower rates of recurrence. Defining the role of cytology post-TMT is warranted as an available and inexpensive biomarker to guide survivorship and salvage protocols.

\section{UP-37}

Prehabilitation body mass index (BMI) target for complication risk reduction following radical cystectomy

Louise C. McLoughlin', Wassim Kassouf', Rodney H. Breau', Adrian Fairey ${ }^{4}$ Agnihotram V. Ramanakumar ${ }^{5}$, Afsar Salimi ${ }^{6}$, Eric Hyndman', Darrel E. Drachenberg ${ }^{8}$, Jonathan Izawa ${ }^{9}$, Bobby Shayegan ${ }^{10}$, Jean-Baptiste Lattouf ${ }^{11}$, Michele Lodde ${ }^{12}$, Ricardo A. Rendon ${ }^{13}$, D. Robert Siemens ${ }^{14}$, Claudio Jeldres ${ }^{15}$, Peter Black ${ }^{16}$, Girish S. Kulkarni ${ }^{1}$

${ }^{1}$ Division of Urology, University of Toronto, Toronto, ON, Canada; ${ }^{2}$ Division of Urology, McGill University, Montreal, QC, Canada; ${ }^{3}$ Division of Urology, University of Ottawa, Ottawa, ON, Canada; ${ }^{4}$ Division of Urology, Cross Cancer Centre, Edmonton, AB, Canada; ${ }^{5}$ Research Institute of McGill University Health Centre, Montreal, QC, Canada; ${ }^{6}$ Centre for Innovative Medicine, Research Institute of McGill University Health Centre, Montreal, QC, Canada; 'Division of Urology, Alberta Health Services, Calgary, AB, Canada; ${ }^{8}$ Section of Urology, Manitoba Cancer Centre, Winnipeg, MB, Canada; ${ }^{9}$ Division of Urology, Western University, London, ON, Canada; ${ }^{10}$ Division of Urology, McMaster Hospital, Hamilton, ON, Canada; ${ }^{11}$ Centre de Recherche du Chum, University of Montreal, QC, Canada; ${ }^{12}$ Division of Urology, Université Laval, Quebec, QC, Canada; ${ }^{13}$ Division of Urology, Capital Health, Halifax, NS, Canada; ${ }^{14}$ Department of Urology, Queen's University, Kingston, ON, Canada; ${ }^{15}$ Division of Urology, Université de Sherbrooke, Quebec, QC, Canada; ${ }^{16}$ Division of Urology, University of British Columbia, Vancouver, BC, Canada; Canadian Bladder Cancer information system (CBCIS) Collaborative, Bladder Cancer Canada

Introduction: Surgical morbidity after radical cystectomy (RC) for muscleinvasive bladder cancer (MIBC) is significant, particularly in patients with an elevated body mass index (BMI). Prehabilitation is a multimodal, pre- operative optimization program reported to reduce complications after major cancer surgery. Nutritional optimization and weight loss are the cornerstone of prehabilitation, especially for patients with an above-normal BMI. This study proposes identifying the optimum BMI range for overweight and obese patients undergoing RC to aim for during prehabilitation and to reduce their risk of perioperative ( $\leq 90$ days) surgical morbidity. Methods: Data were extracted from the Canadian Bladder Cancer information system for this study. A retrospective analysis was performed on 589 patients who underwent RC for MIBC and NMIBC. Perioperative $(\leq 90$ days) Complications were classified by type and severity according to the Clavien-Dindo classification (CDC). Logistic regression analysis was performed to determine the association between patient BMI at time of $\mathrm{RC}$ and the risk of surgical morbidity.

Results: In this cohort, 168 (29\%), 225 (38\%), 125 (21\%), and $71(12 \%)$ patients had a normal, overweight (OW), obese (OB), and morbidly obese (MO) BMI, respectively. Overall, complications occurred in $233(40 \%)$ patients; those with an OW BMI had significantly more CDC grade $3 \mathrm{~b}$ complications (OW: $n=14(54 \%), p=0.37$ ), while those with an MO BMI had significantly more grade 4 complications (MO: $n=4(80 \%), p<0.001)$. Grade $3 b(p=0.037)$ and $4(p<0.0001)$ complications occurred more frequently in those with an above-normal BMI on analysis of the sub-cohort with CDC information available. The most reported complications were ileus in $71(12 \%)$, wound infection in $48(8 \%)$, urine leak in $30(5 \%)$, and fascial dehiscence in $22(4 \%)$ patients. Patients in the OW and $\mathrm{MO}$ category were $1.8(95 \%$ confidence interval $[\mathrm{Cl}] 1.2-2.9, \mathrm{p}=0.008)$ and $3.6(95 \% \mathrm{Cl} 2.0-6.6, \mathrm{p}<0.0001)$ times more likely to develop complications. The risk of urine leak was significantly higher in both OW (odds ratio $[\mathrm{OR}] 5.34,95 \% \mathrm{Cl} 1.16-24.4, \mathrm{p}=0.031)$ and $\mathrm{MO}(\mathrm{OR} 5.32,95 \% \mathrm{Cl}$ $1.0-28.59, \mathrm{p}=0.051)$ categories. A similar trend was observed in $\mathrm{MO} \mathrm{BMI}$ patients, who were at significantly increased risk of both wound infection (OR 5.98, 95\% Cl 2.29-15.61, $\mathrm{p}<0.0001$ ) and fascial dehiscence (OR $5.61,95 \% \mathrm{Cl} 1.23-25.56, \mathrm{p}=0.026)$.

Conclusions: This study demonstrates the feasibility of using a national bladder cancer database to record and evaluate RC complications. Patients with an OW and MO BMI were at significantly increased risk of perioperative morbidity following RC. Patients with an OW and MO BMI could substantially reduce their risk of surgical morbidity by lowering their BMI by one category during prehabilitation.

\section{UP-38}

Pathological upstaging in patients undergoing radical cystectomy for clinically localized de novo muscle-invasive bladder cancer Waleed Shabana', Asmaa Ismail', Hazem Elmansy', Walid Shahrour', Owen Prowse ${ }^{1}$, Ahmed Kotb

${ }^{1}$ Urology, Northern Ontario School of Medicine, Thunder Bay, ON, Canada

Introduction: The aim of this study was to compare the concordance of pathological staging between transurethral resection of bladder tumor (TURBT) and radical cystectomy. The secondary aim was to look for the final pathological outcome of patients with clinically localized muscleinvasive bladder carcinoma (MIBC.

Methods: Fifty-nine patients underwent radical cystectomy for organ-confined de novo T2 MIBC over a two-year period. Clinical and pathological characteristics of TUTBT and cystectomy specimen were collected and statistically analyzed. The possibility of the delay in receiving neoadjuvant chemotherapy was tested as a possible contributing factor to final pathological outcome.

Results: Of the 59 patients, $80 \%$ were male and $20 \%$ were female, with mean age $67+8.8$ years. All patients had preoperative T2 transitional cell carcinoma. Upstaging was noted in 59\% (T3 in $27.1 \%$ and T4 in $32.2 \%$ ). Thirty-six percent of all patients had node-positive disease. Prostate adenocarcinoma was incidentally discovered in 20 (34\%) patients. Among patients who had neoadjuvant chemotherapy, $11(78.5 \%)$ had T2 disease, while only $3(21.6 \%)$ had T3/T4 disease $(\mathrm{p}=0.001)$.

Conclusions: Pathological upstaging was detected in more than half of patients undergoing radical cystectomy for clinically localized disease. Neoadjuvant chemotherapy was underused and was significantly correlated with no upstaging. 
UP-39

\section{Infantile Bacillus Calmette-Guérin vaccination and bladder cancer incidence: Preliminary analysis}

Sabrina Trigo', Kaitlin Gonzalez', Livio Di Matteo², Asmaa Ismail', Waleed Shabana', Hazem Elmansy', Walid Shahrour ${ }^{1}$, Owen Prowse', $\underline{\text { Kotb }^{1}}$

${ }^{1}$ Urology, Northern Ontario School of Medicine, Thunder Bay, ON, Canada; ${ }^{2}$ Cconomics, Lakehead University, Thunder Bay, ON, Canada Introduction: The Bacillus Calmette-Guérin (BCG) vaccine has long been used for the prevention of tuberculosis (TB) around the world. BCG is also used as an immunotherapy agent for the treatment of non-muscleinvasive urinary bladder cancer. This preliminary analysis aims to analyze the available data for infantile BCG vaccination worldwide with the future development of bladder cancer.

Methods: Studies were identified by a formal literature search of MEDLINE and Cochrane Central. Preliminary data analysis was conducted on publicly accessible data summarizing the impact of gender, BCG vaccination, and socioeconomic effects on crude and age-standardized rates of bladder cancer.

Results: As part of our analysis, preliminary regression models demonstrated BCG vaccination status, gender, and socioeconomic status to have statistically significant effects on crude and age-standardized rates of bladder cancer incidence. BCG vaccination was associated with a $35-37 \%$ lower age-standardized rate of bladder cancer incidence.

Conclusions: There is very little literature examining the relationship between prior BCG vaccination and rates of bladder cancer incidence. Our limited data analysis indicates that a relationship does exist between infantile BCG vaccination and later bladder cancer development, although extensive future investigation is needed in this area.

\section{UP-40}

\section{Clinical utility of serial creatinine measurements in patients} undergoing radical cystectomy for urothelial cell carcinoma Christopher Bitcon ${ }^{1}$, Jenna Coles ${ }^{1}$, Stewart Whalen ${ }^{1}$, Ricardo A. Rendon', Gregory G. Bailly', David G. Bell', Ashley R. Cox' ${ }^{1}$, Jon Duplisea', Karthik Tennankore', Ross Mason

${ }^{1}$ Department of Urology, Dalhousie University, Halifax, NS, Canada Introduction: Routine daily blood testing, including serial creatinine $(\mathrm{s} \mathrm{Cr})$ measurement, is commonly performed in postoperative patients. However, prolonging serial measurements may lead to unnecessary healthcare spending, prolonged hospital stay, and patient discomfort and anxiety. We examined the rates of clinical actions (CAs) as a result of $\mathrm{s} C \mathrm{r}$ values, as well as postoperative creatinine trends in order to assess the utility of sCr measurements on radical cystectomy (RC) patients.

Methods: We performed a retrospective chart review using our institution's database on all patients who underwent a RC for urothelial carcinoma (UC) from 2009-2019. Preoperative and all postoperative inpatient creatinine values were recorded. Minor CAs included fluid boluses, fluid rate changes, and medication changes. Major CAs included operating room take-backs for stent repositioning, nephrostomy tube placement, nephrology consultation, and hemodialysis.

Results: A total of 237 RCs were performed with a total of $2952 \mathrm{sCr}$ measurements. The median number of $\mathrm{sCr}$ measurements per patient was nine and the median length of hospital stay was 10 days. There were 92 minor and 13 major CAs representing 3\% and $0.44 \%$ of sCr measurements, respectively. All major CAs were seen in patients experiencing a complicated postoperative course. The most common minor and major CA were fluid bolus (57) and nephrology consultation (5), respectively. There was no incidence of postoperative dialysis. The median postoperative day with the highest creatinine was day 2 .

Conclusions: SCr measurements remain a clinically valuable tool in postoperative management. Nonetheless, this study suggests that prolonged serial measurement of $\mathrm{sCr}$ is of limited clinical utility. As such, discontinuing $\mathrm{s} C \mathrm{r}$ checks after postoperative day 2 in patients experiencing an uncomplicated postoperative course is safe and may lead to both cost savings and decreased patient discomfort.

\section{UP-41}

Bladder cancer guideline adherence in an Australian tertiary hospital

Oliver Best ${ }^{1,2}$, Henry Wang ${ }^{1}$, Lawrence H. C. Kim ${ }^{1,2}$, Tania Hossack ${ }^{1}$, Manish / Patel 1,2

${ }^{1}$ Department of Urology, Westmead Hospital, Westmead, Australia; ${ }^{2}$ Faculty of Medicine and Health, University of Sydney, Sydney, Australia Introduction: Bladder cancer is the 10th most common cancer worldwide and has the highest lifetime treatment cost of all cancers. Overall, literature demonstrates poor guideline adherence, which may lead to suboptimal patient outcomes.

Methods: Eleven common recommendations identified from four major international guidelines were assessed for all patients with bladder cancer managed at our multidisciplinary uro-oncology cancer clinic between January 1, 2017 and August 31, 2019.

Results: Two hundred and eleven eligible patients were included, of which $79 \%$ were male. A total of 90 transurethral resection of bladder tumor (TURBT) operations were performed in 70 patients over the study period. Complete clearance was achieved in $81 \%$, single-dose postoperative chemotherapy was administered in $47 \%$, and re-resection was performed in $81 \%$ of eligible patients. Postoperative risk-stratification was documented in $21 \%$. Of the patients who received adjuvant intravesical Bacillus Calmette-Guérin (BCG), 83\% completed the full induction course and $39 \%$ completed maintenance treatment. Cystoscopic surveillance for high-risk, non-muscle-invasive bladder cancer (NMIBC) was performed within satisfactory interval in 103 of 151 encounters $(68.2 \%)$, urine cytology was performed in 20 of 151 (13.2\%). Computed tomography of the chest, abdomen, and pelvis staging was performed in $98 \%$ of patients with muscle-invasive disease and neoadjuvant chemotherapy was administered to $71 \%$ of eligible patients, with $100 \%$ receiving cisplatin-based regimens. Adjuvant chemotherapy was administered in $57 \%$.

Conclusions: This is the largest and most comprehensive study investigating bladder cancer guideline adherence in Australia. High adherence rates were found for TURBT criteria, staging imaging, and neoadjuvant chemotherapy, but low for high-risk NMIBC surveillance and use of intravesical chemotherapy.

\section{UP-42}

Opioid use after uro-oncologic surgeries in time of opioids crisis: The Quebec experience

Bruno Turcotte $^{1}$, Michele Lodde', Yves Caumartin', Emma Jacques ${ }^{1}$

${ }^{1}$ Urology, CHU de Québec - Université Laval, Quebec, QC, Canada

Introduction: Opioid overdose and addiction are two contemporary growing problems. As health providers, we have an important role in this problem. Recent literature emphasizes overprescription and its link to opioid-related harms. We conducted a prospective, observational study to find how many opioids uro-oncologic patients really need after surgery in our regional and tertiary center.

Methods: This monocentric study included four oncologic uncomplicated surgeries: open retropubic radical prostatectomy, robot-assisted laparoscopic radical prostatectomy, laparoscopic radical nephrectomy, and laparoscopic partial nephrectomy. The primary outcome was the dose of opioid consumed (in oral morphine-equivalent [MEq]), and secondary outcomes included the amount of overprescribed opioids, the amount of opioid requirement for $80 \%$ of patients, management of unused opioids, and others. Patients were invited to fill out three home questionnaires at zero, one and three months postoperative.

Results: Between October 2019 and August 2020, 60 patients were recruited and included for analysis. Patients used a mean of $30 \mathrm{MEq}$ (six tablets) at home and $80 \%$ of patients used $50 \mathrm{MEq}$ (10 tablets) or less. There was no significant difference in opioid consumption between different surgeries in our study $(p=0.68)$. Patients who consumed less than 27.5 MEq during hospitalization were 4.46 times more prone to consume no opioid at home $(p=0.01)$. A total of $2220 \mathrm{MEq}$ was overprescribed (the equivalent of 444 morphine $5 \mathrm{mg}$ tablets) in only 55 responders. Half of the patients or more kept the remaining opioids at home and only $13 \%$ of them returned the remaining opioids to their pharmacy. 
Conclusions: This study emphasizes the importance of prescribing opioids wisely, as too many tablets are still being prescribed. We should encourage a diminution in total MEq after uro-oncologic surgeries and we could probably decrease it more with optimization of co-analgesia, which was not optimized in this observational study.

\section{UP-43}

Utilization of lymphadenectomy and predictors of lymph node yield in upper tract urothelial carcinoma

Siv Venkat ${ }^{1}$, Patrick Lewicki', Spyridon Basourakos ${ }^{1}$, Douglas Scherr ${ }^{1}$

'Department of Urology, Weill Cornell/New-York Presbyterian Hospital, New York, NY, United States

Introduction: Lymph node (LN) dissection (LND) in high-risk upper tract urothelial carcinoma (UTUC) currently remains underperformed compared to other cancers, partly due to a lack of high-quality evidence. We sought to examine current trends in LND for UTUC, as well as patient factors contributing to the performance and omission of LND. We further sought to examine factors predicting higher LN yields.

Methods: A large, national, hospital-based database (The National Cancer Database) was used to identify all patients from 2004-2016 with nonmetastatic UTUC with urothelial histology who underwent extirpative surgery in the form of either radical nephroureterectomy (RNU) or segmental ureterectomy (SU). Rates of LND were examined, with subgroup analysis performed based on high-risk features (high-grade tumor, tumor over $2 \mathrm{~cm}, \mathrm{pT} 2-4$ disease). Univariate linear regression and multivariate logistic regression were performed to identify clinical variables that predict LN yield.

Results: A total of 30861 patients with UTUC were identified from 2004-2016 who underwent extirpative surgery. Overall, $7377(23.9 \%)$ of these patients had a LND performed and $23484(76.1 \%)$ had no LND (Table 1). Only $27.3 \%$ of high-grade tumors, $24.0 \%$ of tumors over $2 \mathrm{~cm}$, $29.4 \%$ of pT 2 tumors, $32.6 \%$ of pT 3 tumors, and $44.7 \%$ of pT 4 tumors had LND performed (Table 2). LND was more likely to be omitted in older patients, higher Charlson comorbidity index $(\mathrm{CCl})$ score, community hospitals, lower clinical T and N stage disease, lower pathological T stage disease, low-grade tumor, laparoscopic surgery, and with RNU ( $<<0.001$ for all). Patients with LND performed were more likely to have positive margins and lymphovascular invasion present, and to undergo chemotherapy and radiation ( $p<0.001$ for all). In multivariate analysis, predictors of higher LN yield were time from diagnosis to definitive surgery, academic/ research hospital, open surgery, and high-grade disease ( $p<0.05$ for all). The strongest predictor was academic/research hospital (odds ratio [OR] $3.45, p=0.002$ ). Predictors of lower LN yield were increased age, CCl $1 / 2$, and laparoscopic surgery (Table 3 ).

Conclusions: LND remains underperformed by surgeons for high-risk UTUC tumors (high-grade tumor, tumor over $2 \mathrm{~cm}$, pT2-4 disease). LND was more likely to be omitted in older patients with increased comorbidities, lower-stage disease, low-grade tumor, with laparoscopic approaches, with RNU, and at community hospitals. Predictors of higher LN yield were time from diagnosis to definitive surgery, academic/research hospital, open surgery, and high-grade disease, with the strongest predictor being academic/research hospital setting.

\begin{tabular}{|c|c|c|c|}
\hline & $\begin{array}{c}\text { LND } \\
\text { performed }\end{array}$ & $\begin{array}{l}\text { No LND } \\
\text { performed }\end{array}$ & $\mathbf{p}$ \\
\hline Age & $\begin{array}{c}71.0 \\
(62.0-78.0)\end{array}$ & $\begin{array}{c}73.0 \\
(65.0-80.0)\end{array}$ & $<0.0001$ \\
\hline Female sex & $2863(38.8 \%)$ & $9467(40.3 \%)$ & 0.022 \\
\hline \multicolumn{2}{|c|}{ Charlson comorbidity score } & & $<0.0001$ \\
\hline 0 & $5040(68.3 \%)$ & $15069(64.2 \%)$ & \\
\hline 1 & $1609(21.8 \%)$ & $5789(24.7 \%)$ & \\
\hline 2 & $499(6.8 \%)$ & $1834(7.8 \%)$ & \\
\hline$\geq 3$ & $229(3.1 \%)$ & 792 (3.4\%) & \\
\hline Facility type & & & $<0.0001$ \\
\hline $\begin{array}{l}\text { Community cancer } \\
\text { program }\end{array}$ & $413(5.6 \%)$ & $1843(7.9 \%)$ & \\
\hline $\begin{array}{l}\text { Comprehensive } \\
\text { community cancer } \\
\text { program }\end{array}$ & $2270(31.0 \%)$ & $10213(43.7 \%)$ & \\
\hline $\begin{array}{l}\text { Academic/research } \\
\text { program }\end{array}$ & $3794(51.9 \%)$ & $7805(33.4 \%)$ & \\
\hline $\begin{array}{l}\text { Integrated network } \\
\text { cancer program }\end{array}$ & $834(11.4 \%)$ & $3513(15.0 \%)$ & \\
\hline cT stage & & & $<0.0001$ \\
\hline cT0 & $14(0.2 \%)$ & $70(0.3 \%)$ & \\
\hline cTa & $921(12.5 \%)$ & 4569 (19.5\%) & \\
\hline cTis & $141(1.9 \%)$ & $497(2.1 \%)$ & \\
\hline cT1 & $980(13.3 \%)$ & $3456(14.7 \%)$ & \\
\hline cT2 & $352(4.8 \%)$ & $1048(4.5 \%)$ & \\
\hline cT3 & 807 (10.9\%) & $2,159(9.2 \%)$ & \\
\hline cT4 & $188(2.5 \%)$ & $310(1.3 \%)$ & \\
\hline cTX & $3466(47.0 \%)$ & $9983(42.5 \%)$ & \\
\hline cN stage & & & $<0.0001$ \\
\hline cNO & $4362(59.1 \%)$ & 16669 (71.0\%) & \\
\hline cN1 & $373(5.1 \%)$ & $140(0.6 \%)$ & \\
\hline $\mathrm{cN} 2$ & $330(4.5 \%)$ & $154(0.7 \%)$ & \\
\hline cN3 & $15(0.2 \%)$ & $13(0.1 \%)$ & \\
\hline cNX & 1997 (27.1\%) & $5861(25.0 \%)$ & \\
\hline pT stage & & & $<0.0001$ \\
\hline pT0 & $42(0.6 \%)$ & $70(0.3 \%)$ & \\
\hline pTa & 1134 (15.4\%) & $5221(22.2 \%)$ & \\
\hline pTis & $162(2.2 \%)$ & $577(2.5 \%)$ & \\
\hline pT1 & $1030(14.0 \%)$ & $3759(16.0 \%)$ & \\
\hline pT2 & 744 (10.1\%) & 1787 (7.6\%) & \\
\hline pT3 & $2403(32.6 \%)$ & $4976(21.2 \%)$ & \\
\hline pT4 & $638(8.6 \%)$ & 789 (3.4\%) & \\
\hline $\mathrm{pX}$ & 1136 (15.4\%) & $5795(24.7 \%)$ & \\
\hline pN stage & & & $<0.0001$ \\
\hline pNo & $4,534(61.5 \%)$ & $7,539(32.1 \%)$ & \\
\hline $\mathrm{pN} 1$ & 733 (9.9\%) & $45(0.2 \%)$ & \\
\hline $\mathrm{pN} 2$ & 835 (11.3\%) & $78(0.3 \%)$ & \\
\hline $\mathrm{pN} 3$ & $44(0.6 \%)$ & $15(0.1 \%)$ & \\
\hline $\mathrm{pNX}$ & $1,140(15.5 \%)$ & $13,676(58.2 \%)$ & \\
\hline $\mathrm{pM}+$ & $158(2.1 \%)$ & $175(0.7 \%)$ & $<0.0001$ \\
\hline
\end{tabular}




\begin{tabular}{|c|c|c|c|}
\hline \multicolumn{4}{|c|}{$\begin{array}{l}\text { UP-43. Table } 1 \text { (cont'd). Demographics of overall cohort } \\
\text { by lymph node dissection status }\end{array}$} \\
\hline & LND performed & $\begin{array}{l}\text { No LND } \\
\text { performed }\end{array}$ & $\mathbf{p}$ \\
\hline Lymph node yield & & & $<0.0001$ \\
\hline 0 & $0(0.0 \%)$ & 22855 (100.0\%) & \\
\hline $1-5$ & $4852(65.8 \%)$ & $0(0.0 \%)$ & \\
\hline $6-10$ & $1325(18.0 \%)$ & $0(0.0 \%)$ & \\
\hline $11-15$ & $621(8.4 \%)$ & $0(0.0 \%)$ & \\
\hline $16-20$ & $279(3.8 \%)$ & $0(0.0 \%)$ & \\
\hline $21-25$ & $132(1.8 \%)$ & $0(0.0 \%)$ & \\
\hline $26-30$ & $75(1.0 \%)$ & $0(0.0 \%)$ & \\
\hline $31-35$ & $31(0.4 \%)$ & $0(0.0 \%)$ & \\
\hline $36-40$ & $23(0.3 \%)$ & $0(0.0 \%)$ & \\
\hline $41-45$ & $11(0.1 \%)$ & $0(0.0 \%)$ & \\
\hline $46-50$ & $16(0.2 \%)$ & $0(0.0 \%)$ & \\
\hline Over 51 & $12(0.2 \%)$ & $0(0.0 \%)$ & \\
\hline High-grade tumor & $6091(82.6 \%)$ & 16231 (69.1\%) & $<0.0001$ \\
\hline Positive margins & $802(11.1 \%)$ & $1512(6.6 \%)$ & $<0.0001$ \\
\hline Surgical approach & & & $<0.0001$ \\
\hline Robotic & 1594 (35.4\%) & $3301(26.1 \%)$ & \\
\hline Laparoscopic & $1270(28.2 \%)$ & $5365(42.3 \%)$ & \\
\hline Open & $1633(36.3 \%)$ & $4003(31.6 \%)$ & \\
\hline LVI present & 1238 (31.9\%) & $2134(20.3 \%)$ & $<0.0001$ \\
\hline XRT given & $202(2.7 \%)$ & $368(1.6 \%)$ & $<0.0001$ \\
\hline Chemotherapy & & & $<0.0001$ \\
\hline No chemo & $5222(72.3 \%)$ & 20404 (88.8\%) & \\
\hline $\begin{array}{l}\text { Underwent } \\
\text { chemo }\end{array}$ & 1939 (26.9\%) & $2305(10.0 \%)$ & \\
\hline $\begin{array}{l}\text { Unknown if } \\
\text { recommended } \\
\text { or administered }\end{array}$ & $58(0.8 \%)$ & $280(1.2 \%)$ & \\
\hline
\end{tabular}

\section{UP-44}

Comparison of radical nephroureterectomy and segmental ureterectomy in upper tract urothelial carcinoma

Siv Venkat ${ }^{1}$, Patrick Lewicki', Spyridon Basourakos ${ }^{1}$, Douglas Scherr ${ }^{1}$

'Department of Urology, Weill Cornell/New-York Presbyterian Hospital, New York, NY, United States

Introduction: Radical nephroureterectomy (RNU) is widely considered the gold standard in high-risk upper tract urothelial carcinoma (UTUC). Segmental ureterectomy (SU) has had increased adoption for high-risk UTUC, particularly for distal ureteric tumors, but its oncological equivalence remains unclear. We sought to examine current trends in RNU and SU for UTUC, patient factors contributing to the performance of SU, compare the rates of lymph node dissection (LND), and examine survival for RNU and SU in high-risk disease.

Methods: A large, national, hospital-based database (The National Cancer Database) was used to identify all patients from 2004-2016 with non-metastatic UTUC with urothelial histology who underwent extirpative surgery (either RNU or SU). Patient factors and rates of LND were compared, with subgroup analysis performed based on high-risk features (high-grade tumor, tumor over 2 cm, pT2-4 disease). Kaplan-Meier methods and log rank testing were used for survival analysis overall and across high-risk subgroups. Results: A total of 30861 patients with UTUC were identified from 2004-2016 who underwent extirpative surgery; 26153 (84.7\%) of these patients underwent RNU and 4708 (15.3\%) underwent SU. SU patients were older, more likely to have surgery at an academic/research hospital,

\begin{tabular}{lc}
\hline $\begin{array}{l}\text { UP-43. Table 2. Number of patients who had lymph node } \\
\text { dissection by high risk feature }\end{array}$ \\
\hline High-risk feature (n) & $\begin{array}{c}\text { Number of patients who had } \\
\text { LND performed (\%) }\end{array}$ \\
\hline High-grade tumor (22 322) & $6091(27.3 \%)$ \\
Tumor over $2 \mathrm{~cm}(20537)$ & $4922(24.0 \%)$ \\
pT2 disease (2531) & $744(29.4 \%)$ \\
pT3 disease (7379) & $2403(32.6 \%)$ \\
pT4 disease (1427) & $638(44.7 \%)$
\end{tabular}

have lower $\mathrm{cT}, \mathrm{cN}, \mathrm{pT}$, and $\mathrm{pN}$ stages, have $\mathrm{pM0}$ disease, have low-grade tumor, be done through open surgery, and have a higher rate of positive margins ( $<<0.001$ for all) (Table 1). LND was more likely to be performed with SU (32.2\%) vs. RNU (23.0\%) ( $<<0.001)$. This was consistent across high-grade tumors, tumors over $2 \mathrm{~cm}$, and pT2-3 disease (Table 2). When patients had a LND, SU had higher nodal yields, with a mean of 6.07 nodes and median of four nodes, vs. RNU, with a mean of 5.69 nodes and median of three nodes. SU was associated with improved overall survival ( $p=0.025)$, with a median survival of 66.1 months for SU and 64.9 months for RNU (Table 3). This was not consistent across high-risk subgroups; there was no significant difference in survival for high-grade tumors $(p=0.14)$, tumors over $2 \mathrm{~cm}(p=0.37)$, $p T 2$ disease $(p=0.072)$, and pT4 disease $(p=0.27)$, with RNU having better survival for $\mathrm{pT} 3$ disease $(\mathrm{p}<0.001)$.

Conclusions: Patients undergoing SU were more likely to be older, have surgery at an academic/research hospital, have lower-stage disease, have low-grade tumor, have open surgery, and had a higher rate of positive margins. LND was more likely to be performed with SU vs. RNU, and this was consistent across high-risk subgroups. When patients underwent LND, SU yielded more nodes on average. SU was associated with improved overall survival compared to RNU for the entire cohort, but there was no statistical difference in survival across high-risk subgroups outside of pT3 disease. While further prospective studies need to be done, SU appears to be non-inferior to RNU in selected high-risk patients.

\section{UP-45}

Disease recurrence patterns in high-risk non-metastatic renal cell carcinoma patients post-nephrectomy with long-term followup Shipra Taneja' ${ }^{7}$, Jen Hoogenes', Anil Kapoor

${ }^{1}$ Department of Surgery, Division of Urology, McMaster University and Urologic Cancer Centre for Research and Innovation, St. Joseph's Healthcare, Hamilton, ON, Canada

Introduction: Guidelines recommend intermediate- and high-risk renal cell carcinoma (RCC) patient monitoring for up to five years; however, some long-term studies have found disease recurrence among the highrisk population after the five-year mark. Our objective was to determine patterns of recurrence among high-risk non-metastatic RCC (HRnmRCC) patients up to15 years post-radical nephrectomy.

Methods: This was a single-center, retrospective chart review of HRnmRCC patients who underwent radical nephrectomy between January 2000 and December 2015. Patients on registered clinical trials for adjuvant therapy or with fewer than three years' followup were excluded. The primary outcome measure was progression-free survival (PFS), with evaluation of prognostic factors, including surgical approach, tumor histology and grade, and margin status.

Results: Fifty patients met the inclusion criteria and had complete data. Mean age at baseline was $70.3( \pm 12.6)$, with a median of 71 years (range 41-97), and 32 were male. Seventy percent had laparoscopic radical nephrectomy, while the rest had open radical nephrectomy. Eight-six percent had clear-cell RCC (cCRCC), four had papillary, two chromophobe, and one sarcomatoid. The most common stages were T3aNXMX and T3aNOMX ( $28 \%$ each), with $90 \%$ having negative margins. A total of 22 patients (16 male) had recurrence, with a median time to recurrence of 28 months (range 7-94). The median age of patients who progressed was 69.5 years (range $41-87$ years) and $91 \%$ had ccRCC. Sixty-eight 


\begin{tabular}{|c|c|c|c|c|}
\hline & \multicolumn{2}{|c|}{ Univariable analysis } & \multicolumn{2}{|c|}{ Logistic multivariable analysis } \\
\hline & OR (95\% Cl) & $\mathbf{p}$ & OR (95\% Cl) & $\mathbf{p}$ \\
\hline Age & $0.959(0.945-0.974)$ & $<0.001$ & $0.974(0.957-0.992)$ & 0.005 \\
\hline Year of diagnosis & $0.949(0.909-0.991)$ & 0.0181 & $1.075(0.978-1.183)$ & 0.132 \\
\hline Time from diagnosis to definitive surgery & $1.011(1.008-1.014)$ & $<0.001$ & $1.011(1.007-1.015)$ & $<0.001$ \\
\hline \multicolumn{5}{|l|}{ Sex } \\
\hline \multicolumn{5}{|l|}{ Charlson comorbidity index } \\
\hline $\mathrm{CCl} 0$ & & Reference & & Reference \\
\hline $\mathrm{CCl} 1$ & $0.323(0.221-0.473)$ & $<0.001$ & $0.396(0.256-0.613)$ & $<0.001$ \\
\hline $\mathrm{CCl} 2$ & $0.373(0.202-0.687)$ & $<0.001$ & $0.372(0.188-0.733)$ & 0.004 \\
\hline $\mathrm{CCl} \geq 3$ & $0.289(0.118-0.710)$ & 0.007 & $0.432(0.165-1.131)$ & 0.087 \\
\hline Academic/ research program & $0.706(0.372-1.341)$ & 0.288 & $3.463(1.605-7.473)$ & 0.002 \\
\hline Integrated cancer network program & $0.0948(0.046-0.195)$ & $<0.001$ & $0.557(0.236-1.317)$ & 0.183 \\
\hline \multicolumn{5}{|l|}{ Surgery type } \\
\hline SU & & Reference & & Reference \\
\hline RNU & $1.226(0.787-1.907)$ & 0.366 & $0.918(0.543-1.550)$ & 0.749 \\
\hline \multicolumn{5}{|l|}{ Surgical approach } \\
\hline Robotic & & Reference & & Reference \\
\hline Laparoscopic & $0.285(0.181-0.449)$ & $<0.001$ & $0.415(0.263-0.656)$ & $<0.001$ \\
\hline Open & 1.638 (1.025-2.617) & 0.039 & 1.766 (1.089-2.864) & 0.021 \\
\hline \multicolumn{5}{|l|}{ Grade } \\
\hline
\end{tabular}

percent of patients had recurrence in the lung, with $50 \%$ having multiple locations. On Kaplan-Meier log rank tests, no factors played a significant role in recurrence.

Conclusions: Our results highlight the importance of followup for HRnmRCC patients beyond five years post-nephrectomy. Our preliminary data suggest that $44 \%(22 / 50)$ can have recurrence up to eight years post-nephrectomy. While this is a small sample size going back to 2000, these preliminary data would suggest that HRnmRCC (T3/T4) should have routine followup beyond five years.

\section{UP-46}

Treatment outcomes with percutaneous thermal ablation for small renal masses

Lennifer Wong ${ }^{1}$, Bjorn Hunter ${ }^{1}$, Christopher Wall ${ }^{2}$, Nicolette R. Sinclair ${ }^{1}$, Jeffery R. Bird ${ }^{1}$, Trustin S. Domes ${ }^{3}$, Kunal Jana ${ }^{3}$

${ }^{1}$ University of Saskatchewan, Saskatoon, SK, Canada; ${ }^{2}$ St. Paul's Hospital, Saskatoon, SK, Canada; ${ }^{3}$ Surgery, University of Saskatchewan, Saskatoon, SK, Canada

Introduction: Thermal ablation (TA) is a well-accepted, minimally invasive treatment option for patients with small renal masses (SRM). As a high-volume TA center, we report our contemporary outcomes data and factors leading to treatment failure.

Methods: A retrospective chart review of patients undergoing TA in Saskatoon, Saskatchewan from 2012-2019 was conducted. Patient and tumor characteristics were analyzed using measures of central tendency. Treatment outcomes and comparison of modalities were analyzed using Wilcoxon test and Chi-squared tests.
Results: The study cohort had a median patient age of 69 years (range3673 ) and median tumor diameter of $2.5 \mathrm{~cm}$ (range 1-6). Ninety-nine microwave ablations (MWAs) and 95 radiofrequency ablations (RFAs) were successfully performed during the study period, with only one attempted procedure not being technically possible. The procedural and post-procedural complication rate (above Clavien-Dindo class 2 ) was $1 \%$. Over a mean followup of 19.9 months (range $0-74$ ), $8.8 \%$ had residual tumor (detected on first imaging study after TA) and $2.6 \%$ developed more remote tumor recurrence. Residual tumor post-TA was associated with larger tumors $(p<0.0001)$ and treatment with MWA $(p=0.039)$, however, MWA-treated tumors were larger $(2.79 \mathrm{~cm}$ vs. $2.41 \mathrm{~cm}, \mathrm{p}=0.02)$ and had a higher biopsy-positive rate for cancer $(56.5 \%$ vs. $47.4 \%, p=0.034)$. In those who required a repeat procedure, $88.2 \%$ were successfully salvaged with additional TA.

Conclusions: TA is a safe and effective treatment option for patients with SRM. Those with residual disease had larger tumors and were more likely to be treated with MWA; however, these patients could be salvaged with more TA in the vast majority of cases. 


\begin{tabular}{|c|c|c|c|}
\hline & $\begin{array}{l}\text { Radical nephro- } \\
\text { ureterectomy }\end{array}$ & $\begin{array}{c}\text { Segmental } \\
\text { ureterectomy }\end{array}$ & $\mathbf{p}$ \\
\hline Age & $72.0(64.0-79.0)$ & $73.0(65.0-80.0)$ & $<0.0001$ \\
\hline Female sex & 10768 (41.2\%) & $1562(33.2 \%)$ & $<0.0001$ \\
\hline \multicolumn{2}{|c|}{ Charlson comorbidity score } & & 0.19 \\
\hline 0 & 17104 (65.4\%) & $3005(63.8 \%)$ & \\
\hline 1 & $6226(23.8 \%)$ & $1172(24.9 \%)$ & \\
\hline 2 & $1958(7.5 \%)$ & $375(8.0 \%)$ & \\
\hline$\geq 3$ & $865(3.3 \%)$ & $156(3.3 \%)$ & \\
\hline Facility type & & & 0.0002 \\
\hline $\begin{array}{l}\text { Community cancer } \\
\text { program }\end{array}$ & $1893(7.3 \%)$ & $363(7.7 \%)$ & \\
\hline $\begin{array}{l}\text { Comprehensive } \\
\text { community cancer } \\
\text { program }\end{array}$ & $10691(41.1 \%)$ & $1792(38.2 \%)$ & \\
\hline $\begin{array}{l}\text { Academic/research } \\
\text { program }\end{array}$ & $9703(37.3 \%)$ & $1896(40.4 \%)$ & \\
\hline $\begin{array}{l}\text { Integrated network } \\
\text { cancer program }\end{array}$ & $3703(14.2 \%)$ & $644(13.7 \%)$ & \\
\hline cT stage & & & $<0.0001$ \\
\hline cT0 & $69(0.3 \%)$ & $15(0.3 \%)$ & \\
\hline cTa & $4442(17.0 \%)$ & $1048(22.3 \%)$ & \\
\hline cTis & $478(1.8 \%)$ & $160(3.4 \%)$ & \\
\hline cT1 & $3697(14.1 \%)$ & $739(15.7 \%)$ & \\
\hline cT2 & $1020(3.9 \%)$ & $380(8.1 \%)$ & \\
\hline cT3 & $2738(10.5 \%)$ & $228(4.8 \%)$ & \\
\hline cT4 & $473(1.8 \%)$ & $25(0.5 \%)$ & \\
\hline cTX & 11667 (44.6\%) & $1782(37.9 \%)$ & \\
\hline cN stage & & & $<0.0001$ \\
\hline cNO & $17634(67.4 \%)$ & $3397(72.2 \%)$ & \\
\hline cN1 & $476(1.8 \%)$ & $37(0.8 \%)$ & \\
\hline cN2 & $470(1.8 \%)$ & $14(0.3 \%)$ & \\
\hline cN3 & $25(0.1 \%)$ & $3(0.1 \%)$ & \\
\hline $\mathrm{cNX}$ & 6747 (25.8\%) & $1111(23.6 \%)$ & \\
\hline pT stage & & & $<0.0001$ \\
\hline pT0 & $95(0.4 \%)$ & $17(0.4 \%)$ & \\
\hline $\mathrm{pTa}$ & $5249(20.1 \%)$ & 1106 (23.5\%) & \\
\hline pTis & $581(2.2 \%)$ & $158(3.4 \%)$ & \\
\hline pT1 & 4137 (15.8\%) & $652(13.8 \%)$ & \\
\hline pT2 & $1774(6.8 \%)$ & 757 (16.1\%) & \\
\hline pT3 & $6657(25.5 \%)$ & 722 (15.3\%) & \\
\hline pT4 & 1389 (5.3\%) & $38(0.8 \%)$ & \\
\hline $\mathrm{pX}$ & $5821(22.3 \%)$ & $1110(23.6 \%)$ & \\
\hline pN stage & & & $<0.0001$ \\
\hline pNO & 9991 (38.2\%) & $2082(44.2 \%)$ & \\
\hline pN1 & $674(2.6 \%)$ & $104(2.2 \%)$ & \\
\hline pN2 & 859 (3.3\%) & $54(1.1 \%)$ & \\
\hline pN3 & $56(0.2 \%)$ & $3(0.1 \%)$ & \\
\hline pNX & $12716(48.6 \%)$ & $2100(44.6 \%)$ & \\
\hline
\end{tabular}

\begin{tabular}{|c|c|c|c|}
\hline \multicolumn{4}{|c|}{$\begin{array}{l}\text { UP-44. Table } 1 \text { (cont'd). Demographics of overall cohort } \\
\text { by surgery type }\end{array}$} \\
\hline & $\begin{array}{l}\text { Radical nephro- } \\
\text { ureterectomy }\end{array}$ & $\begin{array}{l}\text { Segmental } \\
\text { ureterectomy }\end{array}$ & $\mathbf{p}$ \\
\hline $\mathrm{pM}+$ & $320(1.2 \%)$ & $13(0.3 \%)$ & $<0.0001$ \\
\hline Lymph node yield & & & $<0.0001$ \\
\hline 0 & 19706 (77.0\%) & $3149(67.8 \%)$ & \\
\hline $1-5$ & 3945 (15.4\%) & 907 (19.5\%) & \\
\hline $6-10$ & 992 (3.9\%) & $333(7.2 \%)$ & \\
\hline $11-15$ & 479 (1.9\%) & $142(3.1 \%)$ & \\
\hline $16-20$ & $207(0.8 \%)$ & $72(1.5 \%)$ & \\
\hline $21-25$ & $109(0.4 \%)$ & $23(0.5 \%)$ & \\
\hline $26-30$ & $63(0.2 \%)$ & $12(0.3 \%)$ & \\
\hline $31-35$ & $27(0.1 \%)$ & $4(0.1 \%)$ & \\
\hline $36-40$ & $20(0.1 \%)$ & $3(0.1 \%)$ & \\
\hline $41-45$ & $10(0.0 \%)$ & $1(0.0 \%)$ & \\
\hline $46-50$ & $16(0.1 \%)$ & $0(0.0 \%)$ & \\
\hline Over 51 & $11(0.0 \%)$ & $1(0.0 \%)$ & \\
\hline High-grade tumor & 19185 (73.4\%) & 3137 (66.6\%) & $<0.0001$ \\
\hline Positive margins & $1705(6.7 \%)$ & $609(13.6 \%)$ & $<0.0001$ \\
\hline Surgical approach & & & $<0.0001$ \\
\hline Robotic & $4245(29.1 \%)$ & $650(25.0 \%)$ & \\
\hline Laparoscopic & $6089(41.8 \%)$ & $546(21.0 \%)$ & \\
\hline Open & $4234(29.1 \%)$ & $1402(54.0 \%)$ & \\
\hline LVI present & $3016(24.5 \%)$ & $356(17.1 \%)$ & $<0.0001$ \\
\hline XRT given & $459(1.8 \%)$ & $111(2.4 \%)$ & 0.006 \\
\hline Chemotherapy & & & $<0.0001$ \\
\hline No chemo & 21636 (84.5\%) & 3990 (86.9\%) & \\
\hline $\begin{array}{l}\text { Underwent } \\
\text { chemo }\end{array}$ & 3703 (14.5\%) & $541(11.8 \%)$ & \\
\hline $\begin{array}{l}\text { Unknown if } \\
\text { recommended } \\
\text { or administered }\end{array}$ & $277(1.1 \%)$ & $61(1.3 \%)$ & \\
\hline
\end{tabular}

\section{UP-47}

A systematic review and meta-analysis of the significance of body mass index on kidney cancer outcomes

Paul Doan ${ }^{1,2}$, Lawrence H. Kim ${ }^{1,2}$, Yilu He ${ }^{1}$, Howard Lau ${ }^{1,2}$, Henry Pleass ${ }^{1,2}$, Manish I. Patel ${ }^{1,2}$

'Urology, Westmead Hospital, Sydney, Australia; ${ }^{2}$ Surgery, University of Sydney, Sydney, Australia

Introduction: Obesity is a well-known risk factor for kidney cancer incidence. However, several studies have demonstrated more favorable cancer prognosis in patients with elevated body mass index (BMI) conferring a survival advantage, termed the "obesity paradox." We aimed to evaluate the association between $\mathrm{BMI}$ and kidney cancer outcomes (progressionfree survival, cancer-specific survival, and overall survival).

Methods: A computerized systematic search of Medline, Embase, ProQuest, PubMed, and Google Scholar for literature published in English was performed between its inception and December 2018, and Preferred Reporting Items for Systematic Reviews and Meta-Analyses guidelines were used for reporting.

Results: Overall, 34 publications comprising a total of 50717 patients were included in the analysis. The majority assessed the association between BMI and cancer-specific survival. Overweight and obese patients were associated with improved cancer-specific survival compared to patients with normal BMI (hazard ratio [HR] 0.85, 95\% confidence inter- 


\begin{tabular}{lc}
\hline $\begin{array}{l}\text { UP-44. Table 2. Number of patients who had lymph node } \\
\text { dissection performed stratified by high-risk feature }\end{array}$ \\
\hline High-risk feature (n) & $\begin{array}{c}\text { Number of patients who } \\
\text { had LND performed (\%) }\end{array}$ \\
\hline $\begin{array}{l}\text { High-grade tumor } \\
\text { Radical nephroureterectomy } \\
\text { (19 185) }\end{array}$ & $4952(25.8 \%)$ \\
Segmental ureterectomy (3137) & $1139(36.3 \%)$ \\
Tumor over 2 cm & \\
$\begin{array}{l}\text { Radical nephroureterectomy } \\
\text { (18 703) }\end{array}$ & $4294(23.0 \%)$ \\
Segmental ureterectomy (1834) & $628(34.2 \%)$ \\
pT2 disease & \\
Radical nephroureterectomy (1774) & $432(24.3 \%)$ \\
Segmental ureterectomy (757) & $312(41.2 \%)$ \\
pT3 disease & \\
Radical nephroureterectomy (6657) & $2077(31.2 \%)$ \\
Segmental ureterectomy (722) & $326(45.2 \%)$ \\
pT4 disease & $621(44.7 \%)$ \\
Radical nephroureterectomy (1389) & $17(44.7 \%)$ \\
Segmental ureterectomy (38) &
\end{tabular}

val [Cl] 0.79-0.93). A similar trend was demonstrated for progression-free survival (HR 0.68, 95\% Cl 0.59-0.78) and overall survival (HR 0.66, 95\% $\mathrm{Cl}$ 0.55-0.79). On the contrary, the underweight group was associated with inferior cancer-specific survival (HR 2.16, 95\% Cl 1.15-4.04). Main drawbacks limiting the interpretation were the retrospective design in most studies, heterogeneity in study population, BMI classification, and covariates in multivariate analysis.

Conclusions: This is the largest systematic review evaluating the phenomenon of the obesity paradox in kidney cancer outcomes. It demonstrated a favorable effect of BMI on kidney cancer outcomes. However, due to significant heterogeneity of studies, multicenter, prospective studies and further research on the fundamental biological mechanisms are warranted to confirm the significance of BMI on kidney cancer prognosis.

\section{UP-48}

Real world evidence of patient compliance to cabozantinib for metastatic renal cell carcinoma: A single Canadian center experience

Laehoon Kim ${ }^{1}$, Camilla Tajzler², Anil Kapoor ${ }^{2}$

'Faculty of Health Sciences, McMaster University, Hamilton, ON, Canada; ${ }^{2}$ Department of Urology, McMaster University, Hamilton, ON, Canada Introduction: The diagnosis of kidney cancer is often incidental, with $17 \%$ of renal cell carcinomas (RCCs) presenting with distant metastases. Multicenter clinical trials, such as CABOSUN and METEOR, demonstrated efficacy of cabozantinib in metastatic RCC patients. ${ }^{1,2}$ The associated toxicities of cabozantinib often limit patient adherence, which results in suboptimal antitumor activity. This study aimed to evaluate patient adherence to cabozantinib and outline the associated toxicities in daily clinical practice at a single cancer center.

Method: We conducted a retrospective chart review of metastatic RCC patients at McMaster University between 2018 and 2020 that received cabozantinib at any-line therapy with at least three-month followup. Outcomes included time to discontinuation, time to dose reduction, progression-free survival, and overall toxicity profile of cabozantinib. Results: A total of 28 patients were evaluated, with a mean age of $59.4 \pm 8.3$ years and a median time of exposure to cabozantinib of 8.1 months (interquartile range [IQR] 3.3-12.9) (Table 1). Cabozantinib was usually started at the full standard dose of $60 \mathrm{mg}(82 \%)$ and half of the (54\%) patients required dose reductions, with the median time to first dose

\begin{tabular}{lcc}
\hline \multicolumn{2}{l}{ UP-44. Table 3. Median survival stratified by high-risk } \\
feature & $\begin{array}{c}\text { Median survival } \\
\text { (months) }\end{array}$ & p \\
\hline High-risk feature & 52.3 & 0.14 \\
\hline High-grade tumor & 53.1 & \\
$\quad$ Radical nephroureterectomy & & \\
$\quad$ Segmental ureterectomy & 61.2 & 0.37 \\
Tumor over 2 cm & 58.5 & \\
$\quad$ Radical nephroureterectomy & & \\
$\quad$ Segmental ureterectomy & 60.3 & 0.07 \\
pT2 disease & 54.0 & \\
$\quad$ Radical nephroureterectomy & & \\
$\quad$ Segmental ureterectomy & 36.0 & $<0.0001$ \\
pT3 disease & 26.6 & \\
$\quad$ Radical nephroureterectomy & 12.1 & 0.21 \\
$\quad$ Segmental ureterectomy & 19.9 & \\
pT4 disease &
\end{tabular}

reduction at 6.1 months IQR:3.5 - not yet reached) (Fig. 1A). Seven (25\%) patients required treatment discontinuations, with toxicity-induced causes including hypertension $(n=2)$, PPES, and thromboembolic event. Median progression-free survival was 12.8 months (IQR 9.1 - not yet reached) (Fig. 1B). The incidences of previously seen toxicities were lower than clinical trials, except for higher incidences of asymptomatic transaminitis and hypothyroidism.

Conclusions: This study aimed to evaluate the role of cabozantinibinduced toxicities that predispose unwanted dose reductions. These findings provide a benchmark for clinicians to anticipate toxicities and manage them by responding to reversible adverse events in the realworld setting.

References

1. Choueiri TK, Escudier B, Powles T, et al. Cabozantinib vs. everolimus in advanced renal cell carcinoma (METEOR): Final results from a randomized, open-label, phase 3 trial. Lancet Oncol 2016;17:91727. https://doi.org/10.1016/S1470-2045(16)30107-3

2. Choueiri TK, Halabi S, Sanford BL, et al. Cabozantinib vs. sunitinib as initial targeted therapy for patients with metastatic renal cell carcinoma of poor or intermediate risk: The Alliance A031203 CABOSUN Trial. J Clin Oncol 2017;35:591-7. https://doi. org/10.1200/JCO.2016.70.7398

\section{UP-49}

Adrenal pheochromocytoma: Tumor histology correlates with perioperative intensive care stay

Liefei Yao ${ }^{1}$, Luke Witherspoon ${ }^{1}$,Eric Belanger ${ }^{2}$, Neal E. Rowe ${ }^{7}$

${ }^{1}$ Surgery - Urology, University of Ottawa, Ottawa, ON, Canada; ${ }^{2}$ Pathology, University of Ottawa, Ottawa, ON, Canada

Introduction: Pheochromocytomas (PCC) are catecholamine-secreting tumors with the majority arising from the adrenal medulla. The behavior of these tumors is a challenge to predict on the basis of tumor histology. Malignant PCC is only confirmed in the presence of metastatic disease at non-chromaffin sites. Pheochromocytoma of the Adrenal Gland Scale Score (PASS) ${ }^{1}$ is a pathological assessment that helps identify tumors at greater risk of metastatic spread. However, the value of PASS in assessing clinical outcomes apart from metastatic disease is unknown. In our study, we aimed to assess the relationship between PASS and clinical severity of resected adrenal PCC.

Methods: We performed a retrospective chart review on all resected PCC in a tertiary institution from 2008-2018. Pathology slides were reviewed, 


\begin{tabular}{|c|c|c|c|c|c|c|c|c|}
\hline Study or Subgroup & log[Hazard Ratio] & SE & Weight & $\begin{array}{c}\text { Hazard Ratio } \\
\text { IV, Random, } 95 \% \mathrm{Cl}\end{array}$ & & $\begin{array}{r}\text { Hazard } \\
\text { IV, Randor }\end{array}$ & $\begin{array}{l}\text { Ratio } \\
\mathrm{m}, 95 \% \mathrm{Cl}\end{array}$ & \\
\hline Ahmedov2018 & -0.1912 & 0.0708 & $7.3 \%$ & $0.83[0.72,0.95]$ & & - & & \\
\hline awakura2007 & -1.2208 & 0.43 & $0.9 \%$ & $0.29[0.13,0.69]$ & & - & & \\
\hline brookman2010 & -0.1278 & 0.0816 & $6.9 \%$ & $0.88[0.75,1.03]$ & & - & & \\
\hline Byun 2018 & -0.4323 & 0.1603 & $3.9 \%$ & $0.65[0.47,0.89]$ & & $\rightarrow$ & & \\
\hline calle2003 (1) & 0.3343 & 0.1804 & $3.4 \%$ & $1.40[0.98,1.99]$ & & & - & \\
\hline calle $2003(2)$ & 0.4383 & 0.2397 & $2.3 \%$ & $1.55[0.97,2.48]$ & & & - & \\
\hline cho2009 & -1.1394 & 0.6472 & $0.4 \%$ & $0.32[0.09,1.14]$ & & . & & \\
\hline Choi2013 & -0.3769 & 0.2443 & $2.2 \%$ & $0.69[0.42,1.11]$ & & $\rightarrow$ & & \\
\hline eskelimen2017 & 0.0198 & 0.1435 & $4.4 \%$ & $1.02[0.77,1.35]$ & & & & \\
\hline haferkamp2008 & 0.0526 & 0.1791 & $3.4 \%$ & $1.05[0.74,1.50]$ & & . & & \\
\hline hakimi2013 & -0.3079 & 0.1315 & $4.8 \%$ & $0.73[0.57,0.95]$ & & $\rightarrow$ & & \\
\hline ito2017 & -1.8773 & 1.0381 & $0.2 \%$ & $0.15[0.02,1.17]$ & & & & \\
\hline jeon2010 & -0.6349 & 0.3094 & $1.5 \%$ & $0.53[0.29,0.97]$ & & -1 & & \\
\hline komura2011 & -2.3969 & 1.1804 & $0.1 \%$ & $0.09[0.01,0.92]$ & & 一 & & \\
\hline Lee, H 2015 & -0.2797 & 0.213 & $2.7 \%$ & $0.76[0.50,1.15]$ & & $\rightarrow$ & & \\
\hline Lee, Y 2015 & -0.4927 & 0.1664 & $3.7 \%$ & $0.61[0.44,0.85]$ & & $\rightarrow$ & & \\
\hline naya2011 & -2.6173 & 1.8354 & $0.1 \%$ & $0.07[0.00,2.66]$ & & & & \\
\hline Ohno2013 & -0.2182 & 0.0877 & $6.6 \%$ & $0.80[0.68,0.95]$ & & - & & \\
\hline park2013 & 0.1319 & 0.3855 & $1.0 \%$ & $1.14[0.54,2.43]$ & & - & & \\
\hline reeves 2007 (3) & 0.3097 & 0.1154 & $5.4 \%$ & $1.36[1.09,1.71]$ & & & - & \\
\hline rogde 2011 & -0.0513 & 0.0276 & $8.9 \%$ & $0.95[0.90,1.00]$ & & & & \\
\hline schips2004 & -0.0202 & 0.0434 & $8.5 \%$ & $0.98[0.90,1.07]$ & & & & \\
\hline schrader2009 & -0.5276 & 0.1614 & $3.9 \%$ & $0.59[0.43,0.81]$ & & - & & \\
\hline steffens 2013 & -0.3425 & 0.1121 & $5.6 \%$ & $0.71[0.57,0.88]$ & & - & & \\
\hline sung2012 & -0.4155 & 0.1954 & $3.0 \%$ & $0.66[0.45,0.97]$ & & -1 & & \\
\hline waalkes 2010 & -0.0726 & 0.0282 & $8.9 \%$ & $0.93[0.88,0.98]$ & & & & \\
\hline \multirow{2}{*}{\multicolumn{5}{|c|}{$\begin{array}{l}\text { Total }(95 \% \mathrm{Cl}) \\
\text { Heterogeneity: } \mathrm{Tau}^{2}=0.02 ; \mathrm{Chi}^{2}=85.05, \mathrm{df}= \\
\text { Test for overall effect: } Z=3.79(\mathrm{P}=0.0001)\end{array}$}} & & 1 & & \\
\hline & & & & & $\begin{array}{r}0.002 \\
0\end{array}$ & $0.1{ }^{1}$ & $\begin{array}{c}10 \\
\text { Normal BMI }\end{array}$ & 500 \\
\hline $\begin{array}{l}\text { Footnotes } \\
\text { (1) men } \\
\text { (2) Women } \\
\text { (3) Women }\end{array}$ & & & & & & & & \\
\hline
\end{tabular}

UP-47. Fig. 1. Cancer-specific survival based on BMI comparing obese/overweight group with normal BMI control group.

\begin{tabular}{|c|c|c|c|c|c|c|c|c|c|}
\hline Study or Subgroup & $\log [$ Hazard Ratio] & SE & Weight & $\begin{array}{c}\text { Hazard Ratio } \\
\text { IV, Random, } 95 \% \mathrm{Cl}\end{array}$ & & & $\begin{array}{r}\text { Hazard } \\
\text { IV, Rando }\end{array}$ & $\begin{array}{l}\text { d Ratio } \\
\mathrm{m}, 95 \% \mathrm{Cl}\end{array}$ & \\
\hline calle2003 (1) & 0.4194 & 0.215 & $9.2 \%$ & $1.52[1.00,2.32]$ & & & & 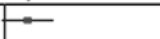 & \\
\hline calle2003 (2) & 0.5188 & 0.2383 & $8.8 \%$ & $1.68[1.05,2.68]$ & & & & & \\
\hline cho2009 & -1.6094 & 0.8212 & $2.5 \%$ & $0.20[0.04,1.00]$ & & & & & \\
\hline Choi2013 & -0.755 & 0.2464 & $8.7 \%$ & $0.47[0.29,0.76]$ & & & $\longrightarrow$ & & \\
\hline haferkamp2008 & 0.1044 & 0.2069 & $9.4 \%$ & $1.11[0.74,1.67]$ & & & & 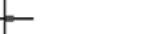 & \\
\hline hakimi2013 & -0.2877 & 0.1771 & $9.9 \%$ & $0.75[0.53,1.06]$ & & & $\rightarrow$ & & \\
\hline jeon2010 & -0.8675 & 0.4047 & $6.1 \%$ & $0.42[0.19,0.93]$ & & & & & \\
\hline Kriegmair2017 & -0.0943 & 0.2855 & $8.0 \%$ & $0.91[0.52,1.59]$ & & & - & - & \\
\hline park2013 & 0.2461 & 0.426 & $5.8 \%$ & $1.28[0.55,2.95]$ & & & - & & \\
\hline parker 2006 & -0.734 & 0.1468 & $10.3 \%$ & $0.48[0.36,0.64]$ & & & $\rightarrow$ & & \\
\hline reeves 2007 (3) & 0.3097 & 0.1154 & $10.8 \%$ & $1.36[1.09,1.71]$ & & & & $\rightarrow$ & \\
\hline steffens 2013 & -0.0619 & 0.129 & $10.6 \%$ & $0.94[0.73,1.21]$ & & & & & \\
\hline Total $(95 \% \mathrm{Cl})$ & & & $100.0 \%$ & $0.87[0.66,1.16]$ & & & & & \\
\hline \multicolumn{5}{|c|}{$\begin{array}{l}\text { Heterogeneity: } \mathrm{Tau}^{2}=0.18 ; \mathrm{Ch}^{2}=60.48, \mathrm{~d} f \\
\text { Test for overall effect: } Z=0.93(P=0.35)\end{array}$} & 0.01 & 0.1 & Obese & \begin{tabular}{|r}
10 \\
Normal BMI
\end{tabular} & 100 \\
\hline \multicolumn{10}{|l|}{$\begin{array}{l}\text { Footnotes } \\
\text { (1) Men } \\
\text { (2) Women } \\
\text { (3) Women }\end{array}$} \\
\hline
\end{tabular}

UP-47. Fig. 2. Cancer-specific survival based on BMI comparing obese vs. normal group. 
Exhibit 2: Non-prostate GU Cancers

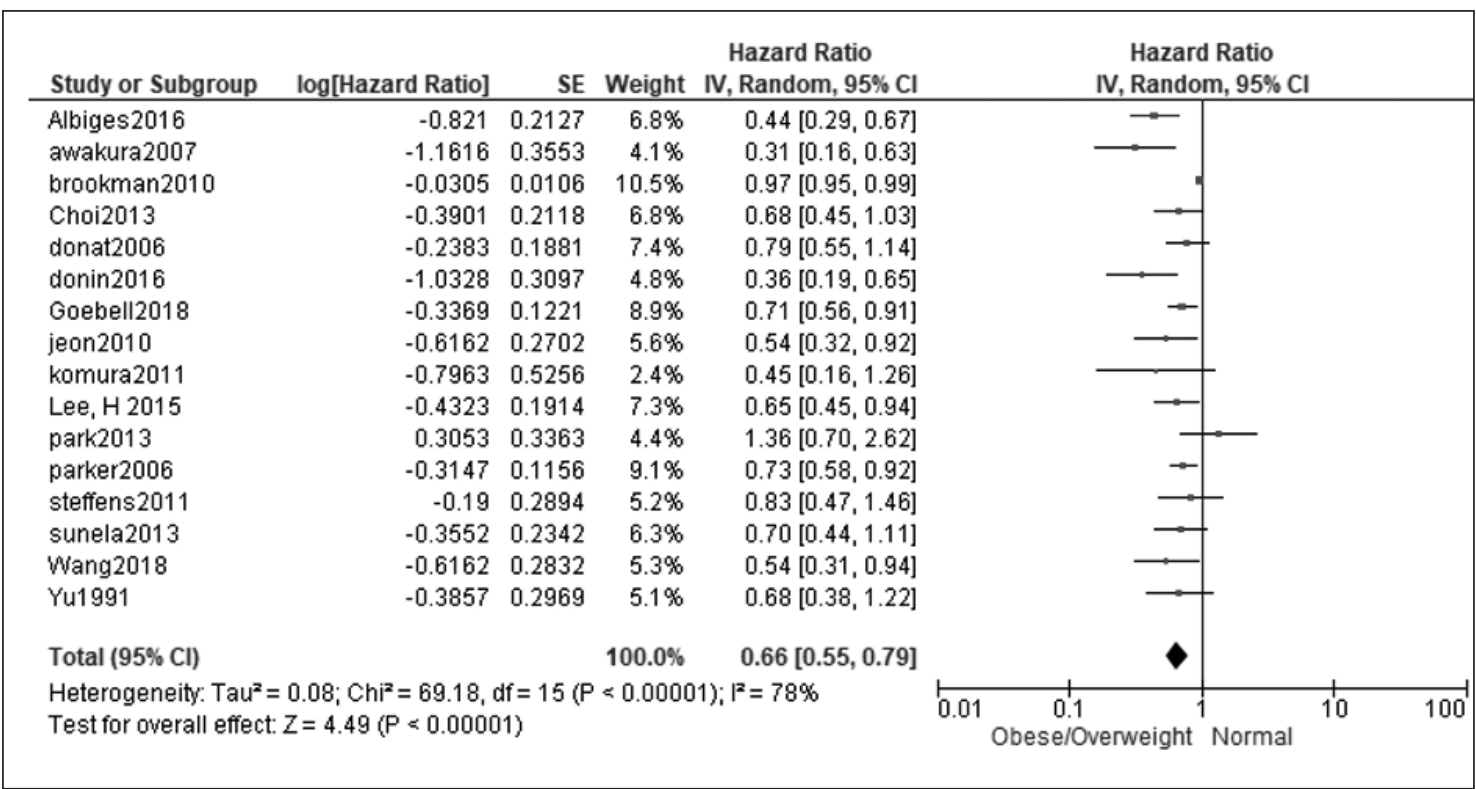

UP-47. Fig. 3. Overall survival based on BMI comparing obese/overweight group with normal BMI control group.

\begin{tabular}{|c|c|c|c|c|c|c|c|c|c|c|}
\hline Study or Subgroup & log[Hazard Ratio] & SE & Weight & $\begin{array}{l}\text { Hazard Ratio } \\
\text { IV, Fixed, } 95 \% \mathrm{Cl}\end{array}$ & & & $\begin{array}{r}\text { Hazard } \\
\text { IV, Fixed, }\end{array}$ & $\begin{array}{l}\text { Ratio } \\
95 \% \mathrm{Cl}\end{array}$ & & \\
\hline Ahmedov2018 & -0.1985 & 0.0628 & $58.7 \%$ & $0.82[0.72,0.93]$ & & & $\square$ & & & \\
\hline Choi2013 & -0.7985 & 0.2242 & $4.6 \%$ & $0.45[0.29,0.70]$ & & & $\rightarrow$ & & & \\
\hline donat2006 & -0.1054 & 0.1901 & $6.4 \%$ & $0.90[0.62,1.31]$ & & & & & & \\
\hline donin2016 & -1.3318 & 0.2555 & $3.5 \%$ & $0.26[0.16,0.44]$ & & & $\rightarrow$ & & & \\
\hline jeon2010 & -1.5141 & 0.5161 & $0.9 \%$ & $0.22[0.08,0.60]$ & & & & & & \\
\hline park2013 & 0.4898 & 0.3695 & $1.7 \%$ & $1.63[0.79,3.37]$ & & & - & & & \\
\hline parker2006 & -0.3285 & 0.1192 & $16.3 \%$ & $0.72[0.57,0.91]$ & & & $\Rightarrow$ & & & \\
\hline steffens 2011 & -0.3285 & 0.3261 & $2.2 \%$ & $0.72[0.38,1.36]$ & & & & & & \\
\hline sunela2013 & -0.4416 & 0.2738 & $3.1 \%$ & $0.64[0.38,1.10]$ & & & 2 & & & \\
\hline Yu1991 & -0.3857 & 0.2969 & $2.6 \%$ & $0.68[0.38,1.22]$ & & & & & & \\
\hline Total $(95 \% \mathrm{Cl})$ & & & $100.0 \%$ & $0.74[0.68,0.82]$ & & & 1 & & & \\
\hline \multirow{2}{*}{\multicolumn{4}{|c|}{$\begin{array}{l}\text { Heterogeneity: } \mathrm{Chi}^{2}=35.43, \mathrm{df}=9(\mathrm{P}<0.0001) ; \mathrm{I}^{2}=75 \% \\
\text { Test for overall effect: } Z=6.16(P<0.00001)\end{array}$}} & & \multirow{2}{*}{0.01} & \multirow{2}{*}{0.1} & 1 & & \multirow[t]{2}{*}{10} & \multirow[t]{2}{*}{100} \\
\hline & & & & & & & Obese & Normal & & \\
\hline
\end{tabular}

UP-47. Fig. 4. Overall survival based on BMI comparing obese vs. normal group.

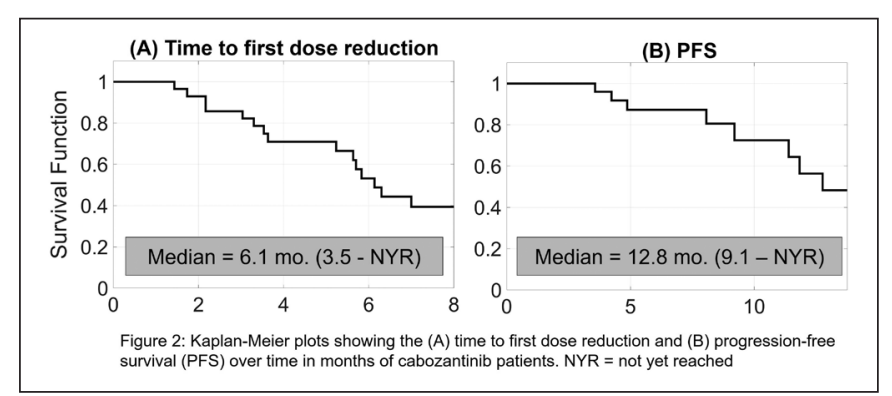

UP-48. Fig. 1. Kaplan-Meier plots showing the (A) time to first dose reduction and $(\boldsymbol{B})$ progression-free survival (PFS) over time in months of cabozantinib patients. NYR: not yet reached.

\begin{tabular}{lccc}
\hline $\begin{array}{l}\text { UP-48. Table 1. Demographic and clinical information } \\
\text { for the retrospective review of cabozantinib patients at } \\
\text { McMaster University }\end{array}$ & N=28 & n & $\%$ \\
\hline Total & Male & $59.4 \pm 8.3$ & \\
\hline Age (y) & 22 & $79 \%$ \\
Sex & Female & 6 & $21 \%$ \\
& 1 & 14 & $50 \%$ \\
\# of metastatic sites & $\geq 2$ & 14 & $50 \%$ \\
& $60 \mathrm{mg}$ & 23 & $82 \%$ \\
\hline
\end{tabular}


assigned a PASS. Demographic information and clinical outcomes were collected for all PCC during the study period. Spearman correlation coefficients were used for comparison of continuous variables.

Results: Sixty-three tumors were identified. Two cases were deemed to be malignant PCC. There was no clear relationship between PASS and patient age, hypertension, cardiac presentation, metanephrine level, peak intraoperative blood pressure, or length of stay. Symptomatic presentations trended toward higher PASS but did not reach statistical significance (6 vs. $4, p=0.47$ ). Median PASS was higher in females than males (6 vs. 2 , $\mathrm{p}=0.017)$. Patients requiring perioperative ICU admission had higher PASS than patients who did not ( 8 vs. $5, p=0.04$ )

Conclusions: This is the first study to use PASS to assess patient outcomes beyond metastatic disease. While metastatic disease is devastating, the more common danger is related to the sequelae of catecholamine excess. We report a novel application of the PASS to confirm a relationship between tumor histology and perioperative outcome in PCC patients While this preliminary work is hypothesis-generating, further studies with larger sample sizes are needed.

\section{Reference}

1. Thompson, LDR. Pheochromocytoma of the Adrenal gland Scaled Score (PASS) to separate benign from malignant neoplasms: A clinicopathologic and immunophenotypic study of 100 cases. Am J Surg Pathol 2002;26:551-66. https://doi.org/10.1097/00000478200205000-00002

\section{UP-50}

Time trends for drug-specific adverse events in patients on sunitinib: Implications for remote monitoring

Lacob Wise', Sophie O'Halloran', Susan Nguyen', Karen Hersey', Neil E. Fleshner

'Division of Urology, University Health Network, University of Toronto, Toronto, ON, Canada

Support: Princess Margaret Cancer Centre's McCain GU BioBank, Queen's University Arts \& Science Undergraduate Research Fund.

Introduction: Sunitinib is a multitargeted receptor tyrosine kinase inhibitor used in the treatment of metastatic renal cell carcinoma (mRCC). Sunitinib can cause many dangerous side effects and patients treated with it require regular, in-person appointments to monitor for adverse events (AEs). Given the current COVID-19 pandemic, it has become increasingly difficult for patients to attend in-person outpatient appointments. This study investigated whether reducing in-person appointments for patients prescribed sunitinib was feasible by a remote monitoring strategy.

Methods: In this retrospective chart review of patients with a diagnosis of mRCC, 167 patients were identified to have received sunitinib during the course of their treatment. The time between initiation of treatment and the first AE was recorded. The AEs included were hypertension, hyperkalemia, liver dysfunction, cardiac event, palmar-plantar eryth-

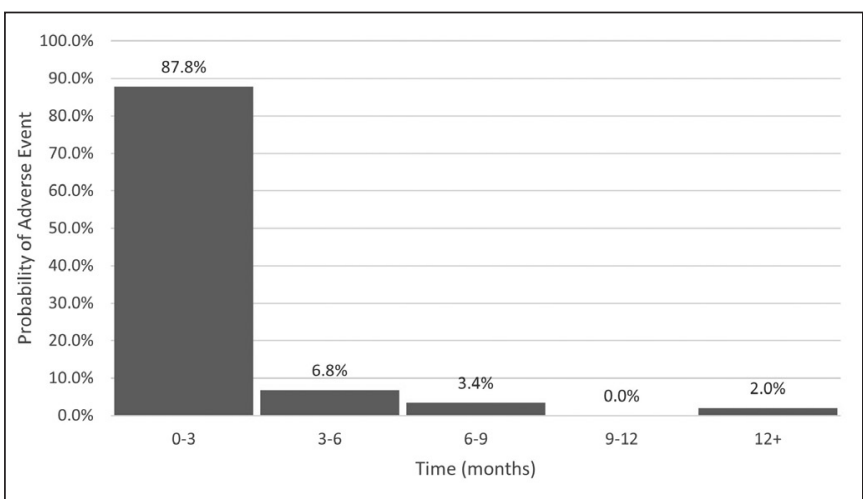

UP-50. Fig. 1. Frequency of duration to first adverse event in patients taking sunitinib for mRCC. rodysesthesia (hand-foot syndrome), diarrhea, and hypothyroidism. The AEs were categorized according to the Common Terminology Crite ria for Adverse Events (CTCAE), version 5. Survival analysis was used to calculate the time to $\mathrm{AE}$.

Results: Of 167 patients identified, 147 experienced an $\mathrm{AE}$ (88\%). Of these patients, cumulative incidence of AEs at three, six, and nine months were $87.8 \%, 94.6 \%$, and $98.0 \%$, respectively (probability of adverse events shown in Fig. 1). The severity of AEs observed were $37.4 \%$ CTCAE grade 1 , followed by $39.4 \%$ grade $2,21.8 \%$ grade 3 , and $1.40 \%$ grade 4 events. There were no grade $5 \mathrm{AEs}$ observed.

Conclusions: Given the current COVID-19 pandemic, decreasing exposure and risk of infection for mRCC patients is essential. We found that patients prescribed sunitinib were most likely to experience the first $\mathrm{AE}$ within three months of treatment and remote monitoring after this time appears appropriate. Furthermore, the AE is likely to be a grade 1 or 2 in severity. We suggest that remote monitoring in the community after three months is feasible, as the likelihood of an AE decreases with time.

\section{UP-51}

Achieving trifecta outcomes early in the robotic-assisted partial nephrectomy learning curve

Dhanika Samaranayake ${ }^{1,2}$, Trent Pattenden ${ }^{1}$, Greg Malone ${ }^{1,2}$, Isaac Thangasamy ${ }^{1}$

${ }^{1}$ Urology, Ipswich Hospital, Ipswich, Queensland, Australia; ${ }^{2}$ Urology, Greenslopes Private Hospital, Greenslopes, Queensland, Australia

Introduction: Achieving trifecta outcomes in robotic-assisted partial nephrectomy (RAPN) is desirable for improved patient safety and clinical outcomes. This study aimed to evaluate achievement of trifecta outcomes for RAPN during the initial learning curve period for an experienced laparoscopic surgeon.

Method: Forty-nine consecutive RAPN cases performed at a tertiary center in Queensland, Australia were reviewed retrospectively. The demographic, histolopathology, and perioperative data was collected, including length of surgery, intraoperative blood loss, and warm ischaemic time (WIT). The trifecta was defined as negative surgical margin, zero perioperative complications, and warm ischemia time (WIT) of less than 20 minutes. Results: The mean operative time was 139 minutes (57-235) and the mean estimated blood loss was $190 \mathrm{ml}$ (20-600). Intraoperative hilar clamping was not performed in five cases. The mean WIT was 16 minutes (8-28). The mean lesion diameter was $28 \mathrm{~mm}(6-52) \mathrm{mm}$. The overall complication rate was $12.2 \%$, of which $>80 \%$ were Clavien-Dindo grade II or less. Positive surgical margins were found in $4.1 \%$ of cases. The mean length of hospital was 4.2 days (2-9) days. Trifecta outcome was achieved in $71.4 \%$ cases. There was no significant difference in incidence of trifecta outcomes between the first 25 cases $(72 \%)$ and the last 24 cases $(71 \%)$. Conclusions: The trifecta outcome of negative surgical margins, no perioperative complications, and a WIT of less than 20 minutes may be achieved early in a surgeon's learning curve with robotic-assisted surgery. To achieve excellent surgical and histological outcomes, a good surgical team and reproducible surgical technique is essential.

\section{UP-52}

Outcomes and prognosticators following radical nephroureterectomy for upper tract urothelial carcinoma Ryan Ramiiawan ${ }^{1}$, Justin Oake', Ryan Sun ${ }^{1}$, David Chung ${ }^{1}$, Robert Wightman', Jasmir (Jay) G. Nayak', Darrel E. Drachenberg', Jeffery W. Saranchuk', Rahul K. Bansal ${ }^{1}$

'Department of Urology, University of Manitoba, Winnipeg, MB, Canada; ${ }^{2}$ Department of Pathology, University of Manitoba, Winnipeg, MB, Canada Introduction: Upper tract urothelial cancer (UTUC) accounts for $5-10 \%$ of all urothelial cancers and approximately $60 \%$ are invasive at diagnosis. Clinical staging remains a challenge, as most ureteroscopic biopsies are often inadequate. The current accepted paradigm for prediction of tumor stage includes a combination of cytology, tumor grade, visual inspection, and radiographic findings. Improvement in prediction of tumor stage would allow for optimal selection of therapeutic options. We analyzed the 
ability of tumor characteristics to predict the presence of muscle-invasive and non-organ-confined (NOC) disease.

Methods: Pathology reports of patients who underwent radical nephroureterectomy (RNU) for UTUC from January 1, 2008 to December 31, 2018 were identified from the Manitoba provincial pathology database. Tumor factors, including grade, size, and location (pelvis/ureter), were identified and correlated with tumor stage using univariable and multivariable analyses.

Results: Of 81 patients who underwent RNU, 74\% had high-grade tumors. Forty-six percent had muscle-invasive disease or higher ( $>\mathrm{pT} 2)$ and $35 \%$ had NOC disease $(\geq \mathrm{pT} 3)$. Presence of high-grade tumor (odds ratio [OR] 12.8, $\mathrm{p}=0.002$ ), size $>45 \mathrm{~mm}$ (OR 3.35, $\mathrm{p}=0.048$ ), and location in both renal pelvis and ureter (OR 3.72, $\mathrm{p}=0.031$ ) were independently associated with $>\mathrm{pT} 2$ disease. Presence of one, two, and three risk factors were associated with rates of $\geq$ pT2 UTUC of $32 \%, 67 \%$, and $71 \%$, respectively. However, only high-grade disease (OR 7.82, $\mathrm{p}=0.010$ ) and size $>45 \mathrm{~mm}$ (OR 2.52, $\mathrm{p}=0.093$ ) were associated with $\geq \mathrm{pT} 3$ disease.

Conclusions: Pathologic-based tumor factors independently predict presence of muscle-invasive and NOC UTUC. Presence of multiple risk factors shows a cumulative effect in the ability to predict $\geq \mathrm{pT} 2$ disease. Further correlation with clinical data and radiographic findings will allow for improved risk-stratification and optimal selection of candidates for neoadjuvant chemotherapy.

\section{UP-53}

Cost-effectiveness analysis of post-orchiectomy management for clinical stage I non-seminoma germ cell testicular cancer

David-Dan Nguyen 1,2,3, Madhur Nayan 3 , Jinyi Zhu', Jane Kim', Christopher J. Sweeney ${ }^{5}$, Steven L. Chang 2,3

${ }^{1}$ Faculty of Medicine, McGill University, Montreal, QC, Canada; ${ }^{2}$ Center for Surgery and Public Health, Brigham and Women's Hospital, Boston, MA, United States; ${ }^{3}$ Division of Urology, Brigham and Women's Hospital, Boston, MA, United States; ${ }^{4}$ Center for Health Decision Science, Harvard T. H. Chan School of Public Health, Boston, MA, United States; ${ }^{5}$ Lank Center for Genitourinary Oncology, Dana-Farber Cancer Institute, Boston, MA, United States

Introduction: The optimal management following initial orchiectomy of testicular cancer patients with a clinical stage (CS) I non-seminomatous germ cell tumor (NSGCT) is controversial. There is a survival equipoise between retroperitoneal lymph node dissection (RPLND), primary chemotherapy, and surveillance following initial orchiectomy. Considering that differences in survival outcomes are marginal, we performed a costeffectiveness analysis to elucidate the tradeoffs and expected values for each management option.

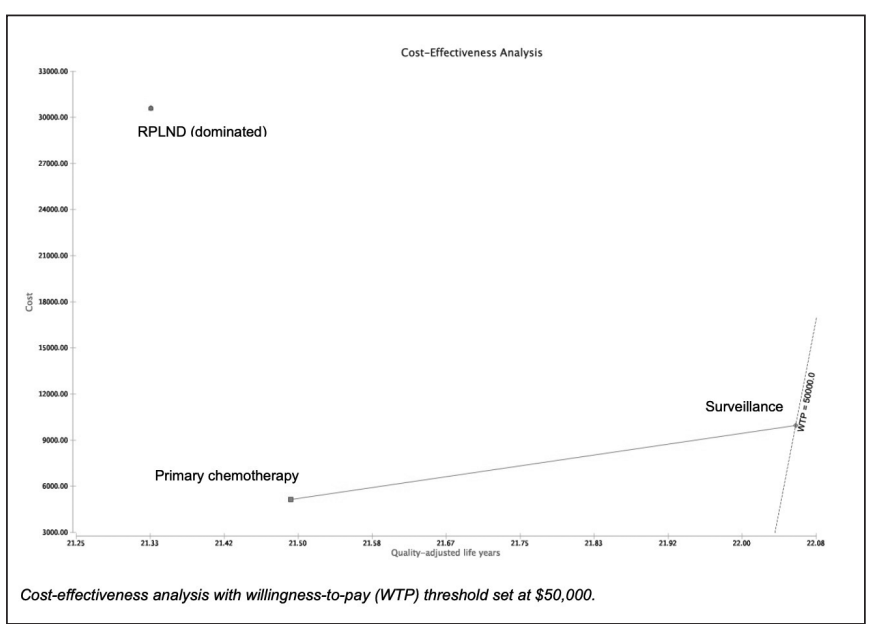

UP-53. Fig. 1. Cost-effectiveness analysis.
Methods: We developed a Markov cohort simulation model that projected overall survival (OS), cancer-specific survival (CSS), quality-adjusted lifeyears (QALYs), and healthcare cost from the payer's perspective with a lifetime time horizon for a cohort of a 33-year-old CS1 NSGCT patients. Disease history, characteristics of relapses, relapse treatment algorithms, and utility values were derived from a comprehensive review of the literature and expert opinion. Costs were determined based on the Premier Hospital Database, a national hospital discharge database representing $20 \%$ of hospitals in the U.S. The model was calibrated and validated against existing literature. A willingness-to-pay (WTP) threshold of \$50 000 per QALY was employed.

Results: In the base case, surveillance was associated with the highest OS, CSS, and QALYs. It was associated with a 94.7\% 15-year OS, a 98.6\% 15-year CSS, and 22.06 QALYs. RPLND was more costly and less effective than both surveillance and primary chemotherapy. The incremental cost-effectiveness ratio associated with surveillance, as compared to primary chemotherapy, was $\$ 1410$ per QALY gained, which is well below the WTP threshold. Results of the cost-effectiveness analysis are summarized in Fig. 1.

Conclusions: We found that surveillance for CS1 NSGCT patients is the optimal strategy compared to RPLND and primary chemotherapy. These findings can inform clinical decision-making, as well as resource allocation.

\section{UP-54}

Surgical outcomes in robotic-assisted partial nephrectomy for imperative vs. elective indications: A propensity score-matched analysis

Io-Lynn Tan ${ }^{1}$, N. Sathianathen ${ }^{1}$, M. Cumberbatch ${ }^{1}$, J. Porter ${ }^{2}$, C. Rogers ${ }^{3}$, P. Dasgupta ${ }^{4}$, A. Mottrie ${ }^{5}$, R. Abaza ${ }^{6}$, K.H. Rha 7 , T. Yuvaraja ${ }^{8}$, D. Parekh ${ }^{9}$, U. Capitanio ${ }^{10}$, R. Ahlawat ${ }^{11}$, S. Rawal ${ }^{12}$, N. Buffi ${ }^{13}$, S. Ananth ${ }^{14}$, K.K. Maes ${ }^{15}$, G. Gautham ${ }^{16}$, F. Porpiglia' ${ }^{17}$, L. Turkeri' ${ }^{18}$, M. Bhandari ${ }^{19}$, D. Moon ${ }^{1}$

'Division of Cancer Surgery, Genitourinary Cancer, Peter MacCallum Cancer Centre, Melbourne, Australia; ${ }^{2}$ Swedish Medical Center, Seattle, WA, United States; ${ }^{3}$ Henry Ford Hospital, Detroit, MI, United States; ${ }^{4}$ DMRC Centre of Transplantation, King's College, London, United Kingdom; ${ }^{5}$ ORSI Academy, Melle, Belgium; ${ }^{6}$ Dublin Methodist Hospital, Ohio, United States; ${ }^{7}$ Yonsei University Health System, Seoul, South Korea; ${ }^{8}$ Kokilaben Dhirubhai Ambani Hospital, Mumbai, India; ${ }^{9}$ University of Miami Health System, Miami, FL, United States; ${ }^{10}$ Urology Clinic, San Raffaele Hospital, Milan, Italy; ${ }^{11}$ Medanta, The Medicity Hospital, New Delhi, India; ${ }^{12}$ Rajiv Gandhi Cancer Institute and Research Centre, New Delhi, India; ${ }^{13}$ Humanitas Research Hospital, Milan, Italy; ${ }^{14} \mathrm{Apollo}$ Hospitals, Greams Lane, Chennai; ${ }^{15}$ Center for Robotic and Minimally Invasive Surgery, Hospital Da Luz, Luz Sáude, Portugal; ${ }^{16}$ Max Institute of Cancer Care, Max Hospital, New Delhi, India; ${ }^{17}$ San Luigi Gonzaga Hospital of Orbassano, Turin, Italy; ${ }^{18}$ Acıbadem Hospitals Group, Istanbul, Turkey; ${ }^{19}$ Vattikuti Foundation, Detroit, MI, United States

Support: Vattikuti Foundation \& Vattikuti Urology Institute, Henry Ford Hospital, Detroit, MI, United States.

Introduction: Partial nephrectomy (PN) is the standard treatment for kidney tumors in imperative settings, defined as situations where there is a single kidney, bilateral renal tumors, and renal impairment. Limited data is available on robotic-assisted PN (RAPN) outcomes for imperative indications. Although recent data has demonstrated RAPN to have reduced complications and length of hospital stay compared to open PN, fewer RAPN are offered for imperative indications, as they are considered highstake scenarios. We sought to compare the perioperative and surgical outcomes in RAPN performed for imperative vs. elective indications.

Methods: We retrospectively interrogated the multinational Vattikuti database of 3801 RAPN cases for elective and imperative indications. Baseline data for age, gender, body mass index (BMI), ASA and PADUA score were examined. Primary outcomes included organ ischemic time, operative time, estimated blood loss, rate of blood transfusions, ClavienDindo complications, conversion to radical nephrectomy (RN), and positive surgical margins. Propensity score-matching was performed to match baseline variables and bivariate analysis used to compare outcomes of interest between groups.

Results: A total of 348 patients (87 imperative vs. 261 elective indications) were included in the final analysis. No significant differences 
were found between groups for ischaemic time (18.9 min vs. $18.6 \mathrm{~min}$, $\mathrm{p}=0.76$ ), operative time (186 min vs. $180 \mathrm{~min}, \mathrm{p}=0.67$ ), estimated blood loss $(226 \mathrm{ml}$ vs. $211 \mathrm{ml}, \mathrm{p}=0.55)$, rate of blood transfusions $(2.5 \% \mathrm{vs}$. $3.4 \%, p=0.99)$, or Clavien-Dindo complications $(\mathrm{p}=0.88)$. There were no conversions to $\mathrm{RN}$ in the imperative group and $6.3 \%(\mathrm{n}=8)$ conversions in the non-imperative group $(\mathrm{p}=0.37)$. Positive surgical margins were seen in $1.2 \%(1 / 87)$ of the imperative group and $4.1 \%$ of the non-imperative group $(1 / 261),(p=0.37)$.

Conclusions: RAPN is feasible and safe for imperative indications, demonstrating comparable, if not superior, outcomes to RAPN performed for elective indications.

\section{UP-55}

Stimulated Raman spectroscopy as a method to improve access and decrease time required to determine renal biopsy adequacy - a pilot study

Miles Mannas ${ }^{1}$, Vasishta Tatapudi ${ }^{2}$, Ming $\mathrm{Wu}^{3}$, Orringer Daniel${ }^{4}$, Samir S. Taneja ${ }^{\top}$

${ }^{1}$ Urology, NYU Langone Health, New York, NY, United States; ${ }^{2}$ Transplant, NYU Langone Health, New York, NY, United States; ${ }^{3}$ Pathology, NYU Langone Health, New York, NY, United States; ${ }^{4}$ Neurosurgery, NYU Langone Health, New York, NY, United States

Introduction: Renal biopsy requires adequate tissue sampling to aid in the investigation of medical renal disease. The adequacy of renal biopsy may be improved by onsite pathologist light microscopy; this evaluation has been shown to increase adequacy rates, decrease the number of biopsies, and increase the number of glomeruli sampled. ${ }^{1}$ Stimulated Raman histology $(\mathrm{SRH})$ is a novel microscopic technique that has created the possibility for rapid, label-free, and high-resolution images of unprocessed tissue, which may be viewed on PACS. ${ }^{2}$
The application of SRH to renal biopsy may provide the benefits of pathological light microscopic evaluation, while also removing the requirement for a renal pathologist to be onsite and decreasing the time required to determine tissue adequacy.

Methods: An 18-gauge core needle biopsy was taken of a radical nephrectomy specimen, remote to a renal mass. Histological images of the fresh, unstained biopsy samples were obtained using a Stimulated Raman spectroscopy microscope using two Raman shifts: $2845 \mathrm{~cm}^{-1}$ and 2930 $\mathrm{cm}^{-1}$. These Stimulated Raman spectroscopic images were then processed to create $\mathrm{SRH}$, which is reminiscent of hematoxylin and eosin, and viewed by a dedicated renal pathologist.

Results: The SRH microscope took seven minutes to produce a highquality image of the renal biopsy (Figs. 1, 2). The seven minutes required to obtain SRH was less then the 23 minutes usually required for pathologist light microscopic evaluation. The biopsy was read by a dedicated renal pathologist without prior SRH training and determined to be diagnostic, which was confirmed by interpretation of the biopsy's H\&E. The pathologist perceived difficulty of SRH interpretation was substantially decreased compared to light microscopic evaluation, and the image quality was considered near hematoxylin and eosin staining.

Conclusions: SRH produces high-quality images that can be rapidly produced and easily interpreted to determine renal biopsy adequacy.

1. Ferrer G, Andeen NK, Lockridge J, et al. Kidney biopsy adequacy. Am J Surg Pathol 2019;43:84-92. https://doi.org/10.1097/ PAS.0000000000001102

2. Orringer DA, Pandian B, Niknafs YS, et al. Rapid intraoperative histology of unprocessed surgical specimens via fibre-laser-based stimulated Raman scattering microscopy. Nat Biomed Eng 2017;1:113. https://doi.org/10.1038/s41551-016-0027

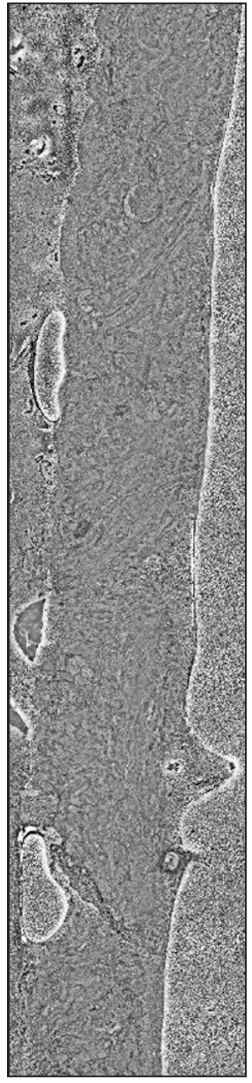

UP-55. Fig. 1. Virtual H\&E image created by clinical stimulated Raman spectroscopy microscope of benign ex-vivo renal biopsy.

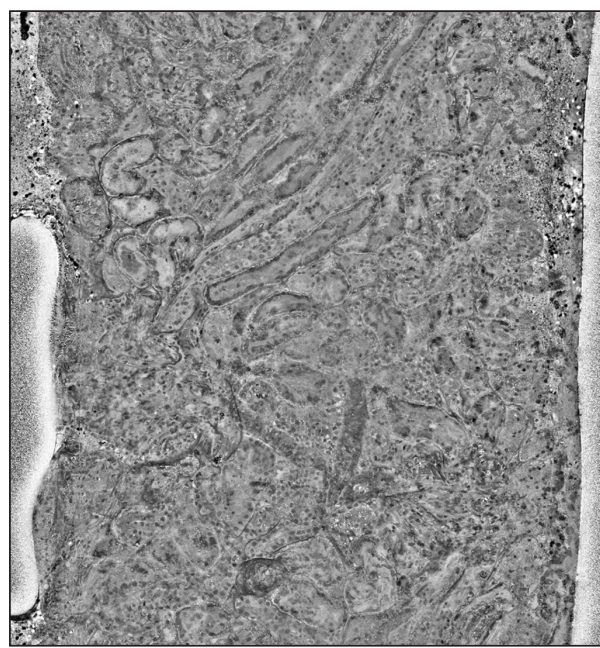

UP-55. Fig. 2. Example of glomeruli from virtual H\&E image created by clinical stimulated Raman spectroscopy microscope of benign ex-vivo renal biopsy from Fig. 1. 